TRANSACTIONS OF THE

AMERICAN MATHEMATICAL SOCIETY

Volume 351, Number 11, Pages 4481-4513

S $0002-9947(99) 02246-1$

Article electronically published on March 24, 1999

\title{
HECKE ALGEBRAS AND COHOMOTOPICAL MACKEY FUNCTORS
}

\author{
NORIHIKO MINAMI \\ Dedicated to Professor Hirosi Toda on his 70th birthday
}

\begin{abstract}
In this paper, we define the concept of the cohomotopical Mackey functor, which is more general than the usual cohomological Mackey functor, and show that Hecke algebra techniques are applicable to cohomotopical Mackey functors. Our theory is valid for any (possibly infinite) discrete group. Some applications to topology are also given.
\end{abstract}

\section{IntRODUCTION}

Hecke algebras have played an important role in the study of the cohomology of groups, more precisely cohomology of subgroups of a fixed (possibly infinite) discrete group $G[40,7,44]$. Because of this, it was a natural hope to find similar applications to the study of more general Mackey functors. Unfortunately, Hecke algebras can act naturally only on the so-called cohomological $G$-functors [46] (since a $G$-functor and a Mackey functor are essentially the same, we call it a cohomological Mackey functor here). Therefore that hope seemed to be an impossible project, at first glance.

Now the purpose of this paper is to realize this hope in the spirit of homotopical algebra $[38,42]$. We define the notion of the cohomotopical Mackey functor (Definition 3.8), which generalizes the notion of the cohomological Mackey functor. Its typical example is the hypercohomology of $G$ with coefficients in a $G$-spectrum $X$ in the sense of [42], which is a natural generalization of the cohomology of the group from the setting of homological algebra to homotopical algebra [38]. (This situation motivated us to use the terminology "cohomotopical Mackey functor.") Then the purpose of this paper is to show that Hecke algebra techniques are appplicable to the cohomotopical Mackey functors also.

More precisely, we fix a commutative ring $k$ and focus our attention on the Hurewicz functor (Definition 2.3)

$$
H_{k}:\left(\mathcal{M}_{k}\right)_{\mathcal{F}}^{\wedge} \rightarrow \mathcal{H}_{k}
$$

where $\left(\mathcal{M}_{k}\right)_{\mathcal{F}}^{\wedge}$ is the cohomotopical Mackey category (Definition 2.35) and $\mathcal{H}_{k}$ is the Hecke category (Definition 2.3). These functors emerge as the cohomotopical Mackey functor is defined to be a $k$-additive functor from $\left(\mathcal{M}_{k}\right)_{\mathcal{F}}^{\wedge}$ and the cohomological Mackey functor is nothing but a $k$-additive functor from $\mathcal{H}_{k}$ (which is Yoshida's theorem [46]).

Received by the editors April 8, 1997.

1991 Mathematics Subject Classification. Primary 55R35, 55P42, 55N91, 19A22, 18G55; Secondary 20C11, 57S17, 55R10.

Key words and phrases. Mackey functor, stable homotopy theory, classifying spaces. 
Our philosophy here is to apply the homological algebra technique (Hecke algebra or Hecke category) to the (stable) homotopical algebra situation (cohomotopical Mackey functors) through the Hurewicz map (Hurewicz functor). Typical questions we would like to consider are: If two objects are equivalent in $\mathcal{H}_{k}$, are they equivalent in $\left(\mathcal{M}_{k}\right)_{\mathcal{F}}^{\wedge}$ ? If there is an idempotent decomposition in $\mathcal{H}_{k}$, is there a corresponding idempotent decomposition in $\left(\mathcal{M}_{k}\right)_{\mathcal{F}}^{\wedge}$ ? Our main results in $\S 4$ answer these questions affirmatively.

This paper is organized as follows. In $\S 2$, we define and investigate basic properties of underlying categories like the Mackey category, the Hecke category, and the cohomotopical Mackey category. In $\S 3$, we define the Mackey functor and other related functors like the cohomotopical Mackey functor. In $\S 4$, we state and prove our main results. For $\S 5$ and $\S 6$, we restrict ourselves to the case $G$ finite and $k=\mathbb{Z}_{p}^{\wedge}$. In $\S 5$, we recall a beautiful identity in the Hecke category by Webb [44] and discuss some applications. As a corollary we get a combinatorial formula involving Möbius functions for cohomotopical Mackey functors over $\mathbb{Z}_{p}^{\wedge}$. In $\S 6$, we give applications to the topology. Here we consider the $p$-completed stable homotopy type of quotient spaces through free $G$-action, especially classifying spaces. We also mention the case of general (not necessarily free) $G$-action. In this case, even though there is no spectra-level combinatorial formula, we do have one at the level of cohomology.

We should emphasize that the philosophy of this paper comes from the homotopy theory. First, our main theorem can be interpreted as a representation theoretical analogue of Nishida's nilpotency theorem on the stable homotopy group of the sphere [36]. Second, we can give a topological proof, which makes use of the Segal conjecture [6], for a special case (i.e. $G$ finite and $k=\mathbb{Z}_{p}^{\wedge}$ ) of the main theorem. Third, the whole project was motivated by the stable splitting of classifying spaces business. Following a conjecture of Priddy, we first proved the corresponding result for classifying spaces (Theorem 6.6), which originally led the author to conjecture our main results in $\S 4$.

If the reader is only interested in such applications to stable splittings of classifying spaces of finite groups (e.g. the Minami-Webb formula), then the reader with a prior knowledge of Yoshida's theorem [46] can jump straight to Lemma 6.8 and avoid the cohomotopical development. Yoshida's theorem [46] itself is reproved in this paper as Theorem 3.7, using our theory.

The original version of this paper was first distributed to experts, including Priddy and Webb, in 1988. (This old version was once again distributed in 1990 as MSRI preprint series 00425-91 [32].) During this period, there has been some progress in the subject $[27,28,41,20,26,21]$. Such updated progress has also occurred in the current version of this paper. First, we have formulated things so that $G$ could be an arbitrary (possibly infinite) discrete group. Second, we do not need the Segal conjecture for our proof of the main theorem, unlike the original version [32]. However, we have also inserted a significantly simplified version of this topological proof based on the Segal conjecture (for the case $G$ finite and $k=\mathbb{Z}_{p}^{\wedge}$ ) in the current paper, because we believe it will give the reader better insight about analogies between algebra and topology.

The author's debt to Professor Stewart Priddy can never be overestimated. Not only did Professor Priddy motivate this work, but also he carefully read a preliminary version of this paper and suggested some improvements. Had it not been for his help, this paper would not have appeared. Also, the author would like 
to thank Professor Haynes Miller for suggesting the use of the Mackey category (Definition 2.1), which turned out to be already given by Linder [25].

\section{UNDERLYING CATEGORIES}

In this section, we define basic underlying categories and discuss their properties, which are needed for our applications. Most of the proofs are straightforward.

Throughout this paper, $G$ is a (possibly infinite) discrete group, and $k$ is a commutative ring, unless otherwise stated.

Definition 2.1. Let $\mathcal{M}$ be the Mackey category defined as follows:

$$
\mathrm{Ob}_{\mathcal{M}}=\{\text { finite } G \text {-sets }\}
$$

where a finite $G$-set is a finite set with a $G$-action.

To define the morphisms, we first consider the set of equivalence classes of the diagrams of the form

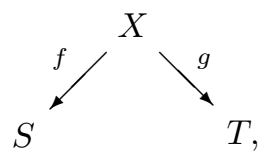

where $X$ is a finite $G$-set, and $f_{S}$ and $f_{T}$ are $G$-maps. Two such objects are set to be equivalent:

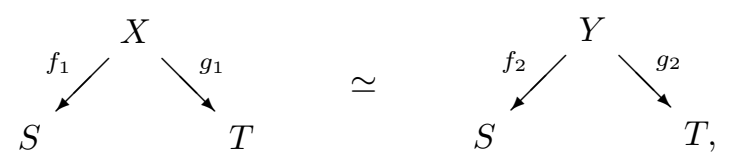

if and only if there is an isomorphism $h: X \rightarrow Y$ of $G$-sets such that $f_{2} \circ h=f_{1}$ and $g_{2} \circ h=g_{1}$. Then the monoid structure is given on representative elements by

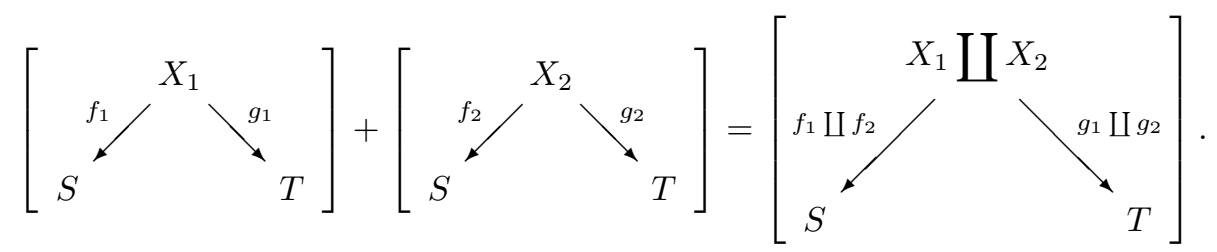

Now $\operatorname{Mor}_{\mathcal{M}}(S, T)$ is defined to be the group completion of this monoid. For $T=S$, the identity $I_{S} \in \operatorname{Mor}_{\mathcal{M}_{k}}(S, S)$ is defined by

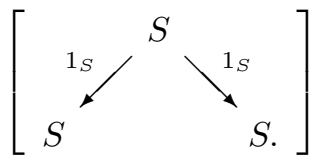

Furthermore, the composition of

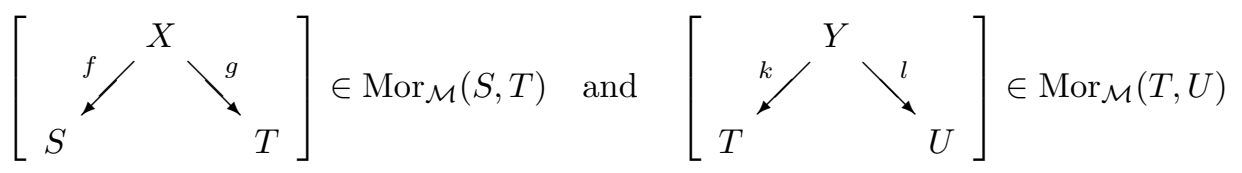


is given on representative elements by the pullback diagram of $G$-sets:

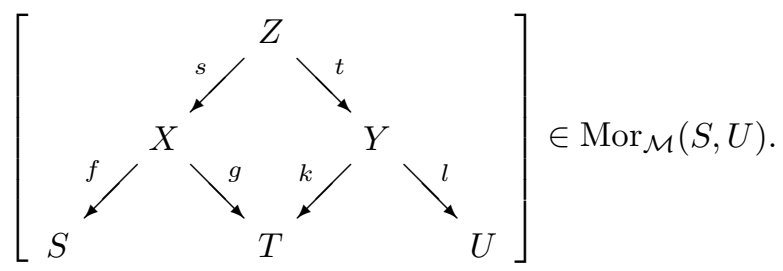

Clearly, this is uniquely extended to a bilinear map

$$
\operatorname{Mor}_{\mathcal{M}}(S, T) \otimes \operatorname{Mor}_{\mathcal{M}}(T, U) \rightarrow \operatorname{Mor}_{\mathcal{M}}(S, U),
$$

which defines the composition.

For any commutative ring $k, \mathcal{M}_{k}$, the Mackey category over $k$, is defined by

$$
\begin{aligned}
\operatorname{Ob}_{\mathcal{M}_{k}} & =\operatorname{Ob}_{\mathcal{M}}, \\
\operatorname{Mor}_{\mathcal{M}_{k}}(S, T) & =\operatorname{Mor}_{\mathcal{M}}(S, T) \otimes_{\mathbb{Z}} k .
\end{aligned}
$$

In particular, $\mathcal{M}_{\mathbb{Z}}=\mathcal{M}$.

Definition 2.2. Given a sequence of finite index subgroups $K \subseteq H \subseteq G$, we define

$$
\begin{gathered}
\operatorname{Res}_{K}^{H} \in \operatorname{Mor}_{\mathcal{M}_{k}}(G / K, G / H), \\
\operatorname{Ind}_{K}^{H} \in \operatorname{Mor}_{\mathcal{M}_{k}}(G / H, G / K), \\
c_{g} \in \operatorname{Mor}_{\mathcal{M}_{k}}\left(G / K^{g}, G / K\right)
\end{gathered}
$$

by

$$
\begin{aligned}
& \operatorname{Res}_{K}^{H}=\left[\begin{array}{c}
{ }_{G / K} \\
1_{G / K}
\end{array}\right] \in \operatorname{Mor}_{\mathcal{M}_{k}}(G / K, G / H), \\
& \operatorname{Ind}_{K}^{H}=\left[\begin{array}{c}
1_{G / H} \\
1_{G / K}
\end{array}\right] \in \operatorname{Mor}_{\mathcal{M}_{k}}(G / H, G / K),
\end{aligned}
$$

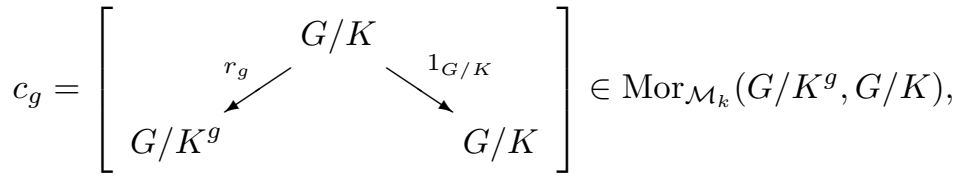

where $p: G / K \rightarrow G / H$ is the canonical projection and $r_{g}: G / K \rightarrow G / K^{g}$ is given by the right multiplication by $g, h K \mapsto h K g=h g K^{g}$.

Definition 2.3. For any commutative ring $k$, we define $\mathcal{H}_{k}$, the Hecke category over $k$, as the category of permutation $G$-modules which are finitely generated over $k$ :

$$
\begin{aligned}
\operatorname{Ob}_{\mathcal{H}_{k}} & =\{\text { finite } G \text {-sets }\}, \\
\operatorname{Mor}_{\mathcal{H}_{k}}(S, T) & =\operatorname{Hom}_{k G}(k S, k T) .
\end{aligned}
$$


We define the Hurewicz functor $H_{k}: \mathcal{M}_{k} \rightarrow \mathcal{H}_{k}$ as follows: On objects, $H_{k}$ is the identity, and on morphisms, $H_{k}$ sends

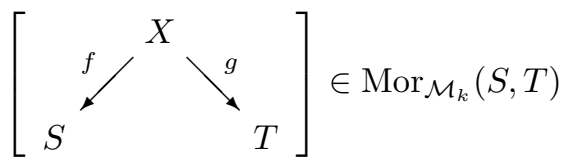

to

$$
\left(s \mapsto \sum_{x \in f^{-1}(s)} g(x)\right) \in \operatorname{Hom}_{k G}(k S, k T) .
$$

Remark 2.4. Suppose $G$ is finite. Then the Mackey category $\mathcal{M}$ is nothing but the full subcategory of the equivariant stable homotopy category $\bar{h} G \mathcal{S} U$ whose objects are the suspension spectra $\Sigma^{\infty} S_{+}$, where $S$ is a finite $G$-set (see [24, v.9] for more detail). This point of view makes the well-definedness of the composition of morphisms in Definition 2.1 trivial.

This point of view also offers another interpretation of the Hurewicz functor $H: \mathcal{M} \rightarrow \mathcal{H}$ for $G$ finite. Actually, $H: \operatorname{Mor}_{\mathcal{M}}(S, T) \rightarrow \operatorname{Mor}_{\mathcal{H}}(S, T)$ is nothing but the evaluation by the 0 -dimensional non-equivariant ordinary reduced homology:

$$
\left\{\Sigma^{\infty} S_{+}, \Sigma^{\infty} T_{+}\right\}_{G} \rightarrow \operatorname{Hom}_{G}\left(\tilde{H}_{0}\left(\Sigma^{\infty} S_{+}\right), \tilde{H}_{0}\left(\Sigma^{\infty} T_{+}\right)\right)=\operatorname{Hom}_{\mathbb{Z} G}(\mathbb{Z} S, \mathbb{Z} T) .
$$

This is essentially the treatment in [43].

Lemma 2.5. Suppose $S=\coprod_{i} S_{i}$ and $T=\coprod_{j} T_{j}$ as finite $G$-sets. Then we have the following commutative diagram with canonical isomorphisms as horizontal arrows:

$$
\begin{array}{ccc}
\operatorname{Mor}_{\mathcal{M}_{k}}(S, T) \stackrel{\cong}{\longrightarrow} \bigoplus_{i, j} \operatorname{Mor}_{\mathcal{M}_{k}}\left(S_{i}, T_{j}\right) \\
H_{k} \downarrow \\
\bigoplus_{i, j} H_{k} \downarrow \\
\operatorname{Mor}_{\mathcal{H}_{k}}(S, T) \stackrel{\cong}{\longrightarrow} \bigoplus_{i, j} \operatorname{Mor}_{\mathcal{H}_{k}}\left(S_{i}, T_{j}\right) .
\end{array}
$$

When $G$ is finite, the upper isomorphism in the following lemma may be interpreted as the $G$-self-duality theorem of Wirthmüller [45] through the equivariant stable homotopy theoretical interpretation in Remark 2.4.

Lemma 2.6. For any finite $G$-sets $S, T$, and $U$, we have the following commutative diagram with canonical isomorphisms as horizontal arrows:

$$
\begin{array}{ccc}
\operatorname{Mor}_{\mathcal{M}_{k}}(S, T \times U) \stackrel{\cong}{\longrightarrow} \operatorname{Mor}_{\mathcal{M}_{k}}(S \times T, U) \\
H_{k} \downarrow \\
\operatorname{Mor}_{\mathcal{H}_{k}}(S, T \times U) \stackrel{H_{k} \downarrow}{\cong} \operatorname{Mor}_{\mathcal{H}_{k}}(S \times T, U) .
\end{array}
$$

Proof. The upper horizontal isomorphism is given by

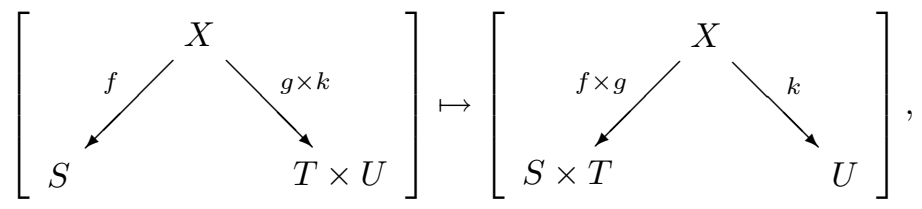


and the lower horizontal isomorphism is given by

$$
\begin{aligned}
& h \in \operatorname{Hom}_{k G}(k S, k T \times U) \\
& \quad \mapsto\left(\rho \otimes 1_{k U}\right) \circ\left(1_{k T} \otimes \epsilon\right) \circ\left(h \otimes 1_{k T}\right) \in \operatorname{Hom}_{k G}(k S \times T, k U),
\end{aligned}
$$

where $\rho: k T \times T \rightarrow k$ is characterized by $\rho(t, t)=1$ and $\rho\left(t, t^{\prime}\right)=0$ if $t \neq t^{\prime}$, and $\epsilon: k U \times T \rightarrow k T \times U$ is induced by the interchange of $U$ and $T$.

To check the commutativity of this diagram, notice that the middle square in

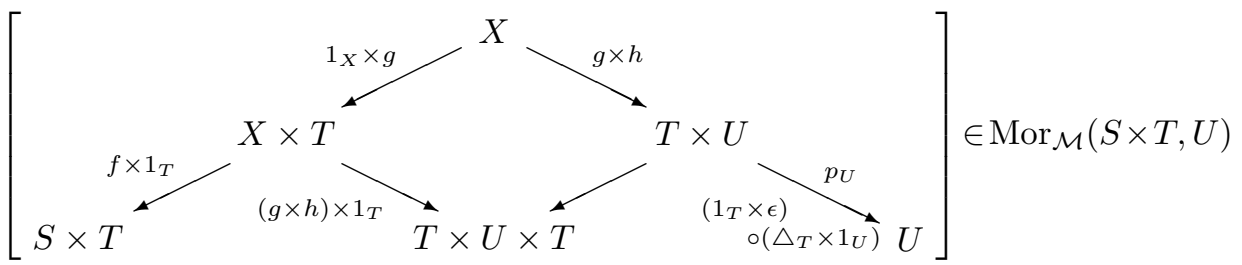

is a pullback diagram, where $\delta_{T}: T \rightarrow T \times T$ is a diagonal map, $\epsilon: U \times T \rightarrow T \times U$ is the interchange of $U$ and $T$, and $p_{U}$ is the projection onto $U$.

Definition 2.7. Given finite $G$-sets $S, T, U$ and $V$, the exterior pairing is defined by

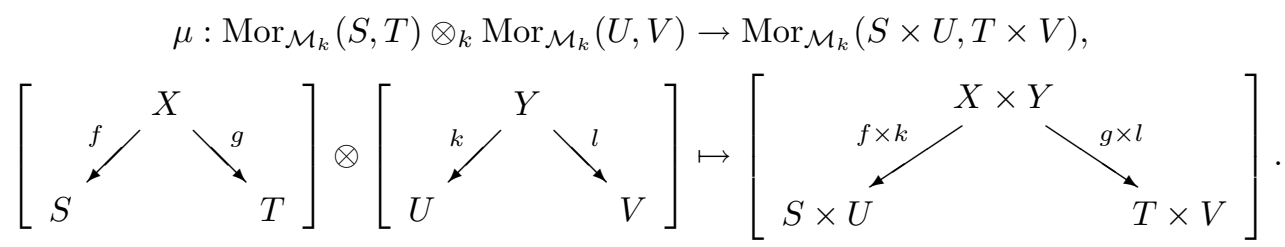

Definition 2.8. Let $f: G_{1} \rightarrow G_{2}$ be a group homomorphism, and let $\mathcal{M}_{k}\left(G_{1}\right)$ and $\mathcal{M}_{k}\left(G_{2}\right)$ be Mackey categories of $G_{1}$ and $G_{2}$, respectively. Then we may define a functor

$$
f^{*}: \mathcal{M}_{k}\left(G_{2}\right) \rightarrow \mathcal{M}_{k}\left(G_{1}\right)
$$

by regarding a finite $G_{2}$-set and a diagram of finite $G_{2}$-sets as a finite $G_{1}$ set and a diagram of finite $G_{1}$-sets, respectively, through $f: G_{1} \rightarrow G_{2}$.

Similarly, $f: G_{1} \rightarrow G_{2}$ induces a functor

$$
f^{*}: \mathcal{H}_{k}\left(G_{2}\right) \rightarrow \mathcal{H}_{k}\left(G_{1}\right)
$$

between the corresponding Hecke categories by regarding a permutation $k G_{2^{-}}$ module (which is finitely generated over $k$ ) as a finitely generated permutation $k G_{1}$-module through $f: G_{1} \rightarrow G_{2}$. Clearly, we have the following commutative diagram:

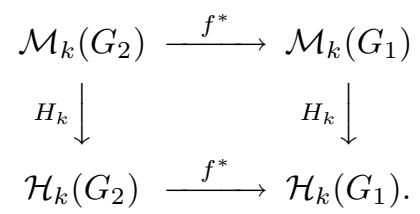

Proposition 2.9. Consider a system of the canonical quotient homomorphisms $\left\{\pi_{N}: G \rightarrow G / N\right\}$, where $N$ runs over finite index normal subgroups of $G$. Then we 
have the following commutative diagram and isomorphisms of categories:

$$
\begin{aligned}
& \lim _{\longrightarrow} N \triangleleft G ; \text { finite index } \mathcal{M}_{k}(G / N) \stackrel{\lim _{N \triangleleft G} \pi_{N}}{\cong} \mathcal{M}_{k}(G) \\
& \lim _{\mathrm{l} \triangleleft G} H_{k} \downarrow \quad H_{k} \downarrow \\
& \lim _{N \triangleleft G ; \text { finite index }} \mathcal{H}_{k}(G / N) \stackrel{\lim _{N \triangleleft G} \pi_{N}}{\cong} \mathcal{H}_{k}(G) \text {. }
\end{aligned}
$$

In particular, given finite $G$-sets $S$ and $T$,

$$
\begin{aligned}
& \lim _{\longrightarrow} N \triangleleft G ; \text { finite index } \operatorname{Mor}_{\mathcal{M}_{k}(G / N)}(S, T) \stackrel{\lim _{N \triangleleft G \pi_{N}}}{\cong} \operatorname{Mor}_{\mathcal{M}_{k}(G)}(S, T) \\
& \stackrel{\lim }{\longrightarrow} \wedge \triangleleft H_{k} \downarrow \quad H_{k} \downarrow \\
& \operatorname{Mor}_{\mathcal{H}_{k}\left(G / N_{0}\right)}(S, T) \stackrel{\pi_{N_{0}}}{\cong} \operatorname{Mor}_{\mathcal{H}_{k}(G)}(S, T) \text {, }
\end{aligned}
$$

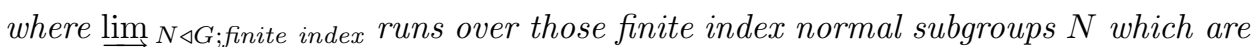
contained in the intersection of all the isotropy subgroups of $S$ and $T$ (this condition enables us to view $S$ and $T$ as finite $G / N$-sets), and $N_{0}$ is any one such normal subgroup.

Proof. Everything is easy to see, except the isomorphism

$$
\operatorname{Mor}_{\mathcal{H}_{k}\left(G / N_{0}\right)}(S, T) \stackrel{\pi_{N_{0}}}{\cong} \operatorname{Mor}_{\mathcal{H}_{k}(G)}(S, T) .
$$

But this easily follows from Lemma 2.5 and the fact that

$$
\operatorname{Mor}_{\mathcal{H}_{k}(G)}(G / H, G / K)=\operatorname{Hom}_{k G}(k G / H, k G / K) \cong k H \backslash G / K .
$$

Remark 2.10. Unlike the Hecke category, we usually cannot find a finite index normal subgroup $N_{0}$ such that $\operatorname{Mor}_{\mathcal{M}_{k}\left(G / N_{0}\right)}(S, T) \stackrel{\pi_{N_{0}}}{\cong} \operatorname{Mor}_{\mathcal{M}_{k}(G)}(S, T)$. A simplest example is $G=\mathbb{Z}, S=T=\mathrm{pt}$.

Definition 2.11. Let $H$ be a finite-index subgroup of $G$. Let $\mathcal{M}_{k}(G)$ and $\mathcal{M}_{k}(H)$ be the Mackey categories over $k$ of $G$ and $H$, respectively. Now define the functor

$$
F_{H}^{G}: \mathcal{M}_{k}(H) \rightarrow \mathcal{M}_{k}(G)
$$

by

$$
\begin{aligned}
& \mathrm{Ob} \mathcal{M}_{k}(H) \rightarrow \mathcal{M}_{k}(G) \\
& S \mapsto G \times_{H} S, \\
& \operatorname{Mor}_{\mathcal{M}_{k}(H)}(S, T) \rightarrow \operatorname{Mor}_{\mathcal{M}_{k}(G)}\left(G \times_{H} S, G \times_{H} T\right) \text {, }
\end{aligned}
$$

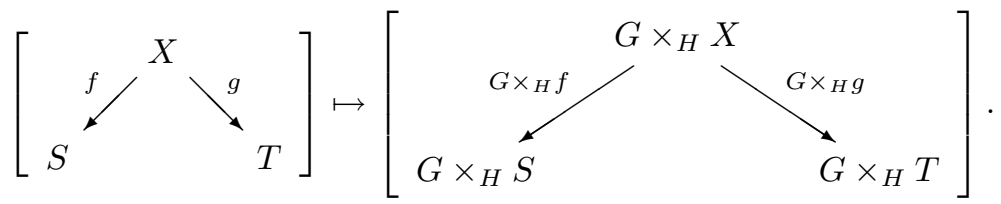

Under the same condition, let $\mathcal{H}_{k}(G)$ and $\mathcal{H}_{k}(H)$ be the Hecke categories over $k$ of $G$ and $H$, respectively. Then, using the induced representation $M \mapsto k G \otimes_{k H} M$, 
we may define the functor $F_{H}^{G}: \mathcal{H}_{k}(H) \rightarrow \mathcal{H}_{k}(G)$ so that the following diagram commutes:

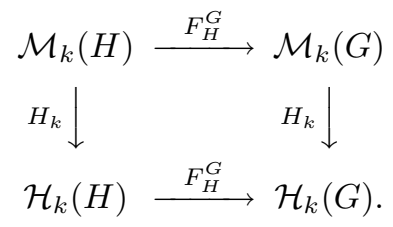

To show that $F_{H}^{G}: \mathcal{M}_{k}(H) \rightarrow \mathcal{M}_{k}(G)$ is really a functor, it suffices to establish

Proposition 2.12. Suppose $H$ is a subgroup of $G$, and

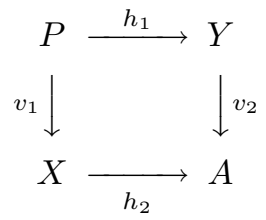

is a pull-back diagram of $H$-sets. Then

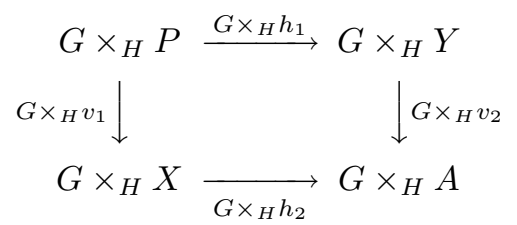

is a pull-back diagram of $G$-sets.

However, this is an immediate consequence of the following easy lemma:

Lemma 2.13. Suppose $H$ is a subgroup of $G, T$ is a $G$-set, $S$ is a $H$-set, and

$$
f: T \rightarrow G \times{ }_{H} S
$$

is a $G$-map. Let $p: G \times_{H} S \rightarrow G / H$ be the obvious canonical projection. Then

1. $(p \circ f)^{-1}(H) \subseteq T$ is an $H$-subset.

2. There is an isomorphism of $G$-sets:

$$
T \cong G \times_{H}\left((p \circ f)^{-1}(H)\right) .
$$

3. Let $\left.f\right|_{(p \circ f)^{-1}(H)}:(p \circ f)^{-1}(H) \rightarrow S\left(=H \times_{H} S \subseteq G \times_{H} S\right)$ be the resulting H-map. Then

$$
f=G \times_{H}\left(\left.f\right|_{(p \circ f)^{-1}(H)}\right) .
$$

Furthermore, up to the identification in 2, the decomposition of $f$ in 3 is unique, i.e. the decomposition in 3 uniquely characterizes $\left.f\right|_{(p \circ f)^{-1}(H)}$.

From this lemma, we also immediately get

Proposition 2.14. Let $H$ be a finite index subgroup of $G$. Then, for any finite $H$-set $S$ and a finite $G$-set $T$, there is a canonical isomorphism

$$
\operatorname{Mor}_{\mathcal{M}_{k}(G)}\left(G \times_{H} S, T\right) \cong \operatorname{Mor}_{\mathcal{M}_{k}(H)}(S, T) .
$$


Remark 2.15. Clearly, the above canonical isomorphism may be completed to the following commutative diagram with canonical horizontal isomorphisms:

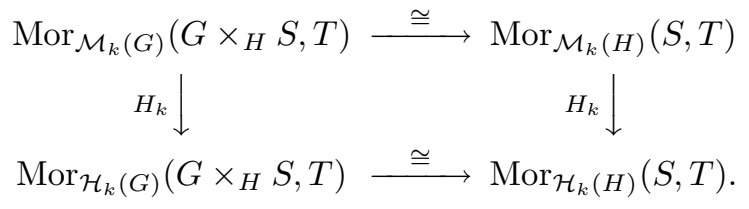

Definition 2.16. For any group $G$, its Burnside ring $A(G)$ is defined to be the Grothendieck ring of finite $G$-sets; its additive structure and multiplicative structures are induced from the disjoint union and the Cartesian product of finite $G$-sets, respectively. For any commutative ring $k$, we set $A(G)_{k}:=A(G) \otimes_{\mathbb{Z}} k$.

We denote the product map by

$$
\begin{aligned}
\mu: A(G)_{k} \otimes_{k} A(G)_{k} & \rightarrow A(G)_{k}, \\
{[X] \otimes_{k}[Y] } & \mapsto[X \times Y] .
\end{aligned}
$$

Furthermore, when $H$ is a subgroup of $G$ and $g$ is an element of $G$, we set $\operatorname{Res}_{H}^{G}: A(G) \rightarrow A(H), \operatorname{Ind}_{H}^{G}: A(H) \rightarrow A(G)$ and $c_{g}: A(H) \rightarrow A\left(H^{g}\right)$ by

$$
\begin{aligned}
\operatorname{Res}_{H}^{G}: A(G) & \rightarrow A(H), \\
{[X] } & \mapsto[X], \\
\operatorname{Ind}_{H}^{G}: A(H) & \rightarrow A(G), \\
{[Y] } & \mapsto\left[G \times_{H} Y\right], \\
c_{g}: A(H) & \rightarrow A\left(H^{g}\right), \\
{[Y] } & \mapsto\left[g^{-1} H \times_{H} Y\right],
\end{aligned}
$$

where we must require that $H$ is of finite index in $G$ to define $\operatorname{Ind}_{H}^{G}$.

Remark 2.17. If $G$ is a compact Lie group, this definition of the Burnside ring does not coincide with tom Dieck's in general.

Definition 2.18. Given a finite $G$-set $S$, we define $A_{G}(S)$ to be the Grothendieck construction of finite $G$-sets over $S$. More precisely, consider diagrams of the form

$$
f: T \rightarrow S,
$$

where $T$ is a finite $G$-set and $f$ is a $G$-map. Two such diagrams $f_{i}: T_{i} \rightarrow S(i=1,2)$ are said to be equivalent if there is an isomorphism of finite $G$-sets $h: T_{1} \rightarrow T_{2}$ such that $f_{1}=f_{2} \circ h$. Furthermore, the monoid structure is given by the disjoint union on representative elements

$$
[g: U \rightarrow S]+[h: V \rightarrow S]=[g \coprod h: U \coprod V \rightarrow S] .
$$

Now $A_{G}(S)$ is defined to be the group completion of this monoid. ( $A_{G}$ was defined and denoted by $\Omega$ as the (Burnside) Green functor in [11, p.303].)

As usual, we set $A_{G}(S)_{k}:=A_{G}(S) \otimes_{\mathbb{Z}} k . A_{G}(S)_{k}$ is equipped with an $A(G)_{k^{-}}$ module structure by

$$
\begin{aligned}
A(G)_{k} \otimes_{k} A_{G}(S)_{k} & \rightarrow A_{G}(S), \\
{[X] \otimes_{k}[f: T \rightarrow S] } & \mapsto\left[f \circ p_{T}: X \times T \rightarrow S\right],
\end{aligned}
$$

where $p_{T}: X \times T \rightarrow T$ is the canonical projection. 
Given a $G$-map $g: S_{1} \rightarrow S_{2}$ between finite sets, its induced map $g_{*}$ is defined by

$$
\begin{aligned}
g_{*}: A_{G}\left(S_{1}\right)_{k} & \rightarrow A_{G}\left(S_{2}\right)_{k}, \\
{\left[f: T \rightarrow S_{1}\right] } & \mapsto\left[g \circ f: T \rightarrow S_{2}\right] .
\end{aligned}
$$

Clearly, this is an $A(G)_{k}$-module map.

Definition 2.19. Given finite $G$-sets $S$ and $T$, we define the exterior pairing by

$$
\begin{aligned}
\mu: A_{G}(S)_{k} \otimes_{k} A_{G}(T)_{k} & \rightarrow A_{G}(S \times T)_{k}, \\
{[g: U \rightarrow S] \otimes[h: V \rightarrow T] } & \mapsto[g \times h: U \times V \rightarrow S \times T] .
\end{aligned}
$$

Clearly, $\mu$ is an $A(G)_{k}$-bilinear map and factors through

$$
A_{G}(S)_{k} \otimes_{A(G)_{k}} A_{G}(T)_{k} \rightarrow A_{G}(S \times T)_{k} .
$$

Definition 2.20. For each finite $G$-set $S$, define a $k$-algebra map

$$
\begin{aligned}
& \triangle_{S}: A(G)_{k} \rightarrow \operatorname{Mor}_{\mathcal{M}_{k}}(S, S), \\
& {[X] \mapsto\left[\begin{array}{c}
p_{S} \\
p_{S} \times X \\
p_{S}
\end{array}\right],}
\end{aligned}
$$

where $p_{S}$ is the projection onto the $S$ factor.

Lemma 2.21. Let $H$ be a finite index subgroup of $G$ and let $S$ be a finite $H$ set. Then we have the following commutative diagram of $k$-algebras and $k$-algebra maps:

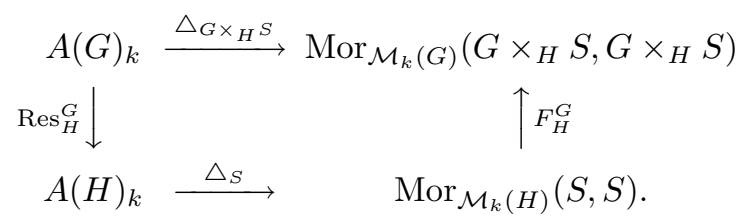

Proof. This easily follows from the following commutative diagram of $G$-sets and $G$ maps:

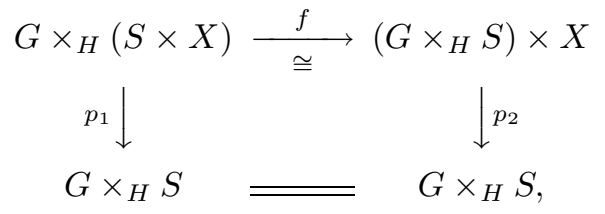

where $X$ and $S$ are finite $G$-set and finite $H$-set, respectively, and

$$
\begin{aligned}
f:(g,(s, x)) & \mapsto((g, s), g x), \\
p_{1}:(g,(s, x)) & \mapsto(g, s), \\
p_{2}:((g, s), x) & \mapsto(g, s) . \quad \square
\end{aligned}
$$

Lemma 2.22. For any finite $G$-sets $S$ and $T$, the following three $A(G)_{k}$-actions on $\operatorname{Mor}_{\mathcal{M}_{k}}(S, T)$ are all well-defined and coincide:

$$
\begin{aligned}
A(G)_{k} & \otimes_{k} \operatorname{Mor}_{\mathcal{M}_{k}}(S, T) \\
& \stackrel{\triangle_{S} \otimes_{k} \operatorname{Mor}_{\mathcal{M}_{k}}(S, T)}{\longrightarrow} \operatorname{Mor}_{\mathcal{M}_{k}}(S, S) \otimes_{k} \operatorname{Mor}_{\mathcal{M}_{k}}(S, T) \\
& \rightarrow \operatorname{Mor}_{\mathcal{M}_{k}}(S, T),
\end{aligned}
$$


where the last map is the composition;

$$
\begin{aligned}
\operatorname{Mor}_{\mathcal{M}_{k}}(S, T) \otimes_{k} A(G)_{k} \\
\quad \stackrel{\operatorname{Mor}_{\mathcal{M}_{k}}(S, T) \otimes_{k} \triangle_{T}}{\longrightarrow} \operatorname{Mor}_{\mathcal{M}_{k}}(S, T) \otimes_{k} \operatorname{Mor}_{\mathcal{M}_{k}}(T, T) \\
\quad \rightarrow \operatorname{Mor}_{\mathcal{M}_{k}}(S, T)
\end{aligned}
$$

where the last map is the composition;

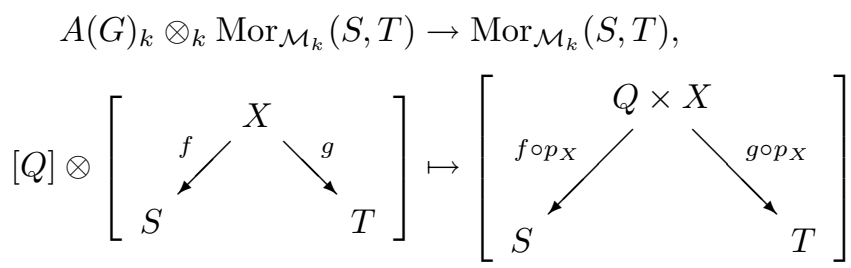

where $p_{X}$ is the projection onto the $X$ factor.

Corollary 2.23. With respect to the $A(G)_{k}$-module structure in Lemma 2.22, the composition of the Mackey category is an $A(G)_{k}$-bilinear map and induces

$$
\operatorname{Mor}_{\mathcal{M}_{k}}(S, T) \otimes_{A(G)_{k}} \operatorname{Mor}_{\mathcal{M}_{k}}(T, U) \rightarrow \operatorname{Mor}_{\mathcal{M}_{k}}(S, U),
$$

for any finite $G$-sets $S, T$ and $U$.

Lemma 2.24. We have the following canonical natural isomorphisms of $A(G)_{k}$ modules:

1. $\operatorname{Mor}_{\mathcal{M}_{k}}(S, T) \cong A_{G}(S \times T)_{k}$.

2. $A_{G}(\mathrm{pt})_{k} \cong A(G)_{k}$.

3. For any finite $G$-sets $S_{i} i=1,2, \cdots, n$, we have

$$
A_{G}\left(\coprod_{i=1}^{n} S_{i}\right)_{k} \cong \bigoplus_{i=1}^{n} A_{G}\left(S_{i}\right)_{k}
$$

4. For any finite index subgroup $H$ of $G$ and finite $H$-set $S$,

$$
\begin{aligned}
A_{H}(S)_{k} & \cong A_{G}\left(G \times_{H} S\right)_{k}, \\
{[f: T \rightarrow S] } & \mapsto\left[G \times_{H} f: G \times_{H} T \rightarrow G \times_{H} S\right] .
\end{aligned}
$$

5. For any finite $G$-set $S=\coprod_{i=1}^{n} G / H_{i}$, we have

$$
A_{G}(S)_{k} \cong \bigoplus_{i=1}^{n} A\left(H_{i}\right)_{k}
$$

Proposition 2.25. For any finite $G$-sets $S$ and $T$, let $S \times T \cong \coprod_{i=1}^{n} G / H_{i}$ be the decomposition into $G$-orbits. Then we have the following commutative diagram with canonical $A(G)_{k}$-module isomorphisms as horizontal arrows:

$$
\begin{array}{ccc}
\operatorname{Mor}_{\mathcal{M}_{k}}(S, T) \stackrel{\cong}{\longrightarrow} \bigoplus_{i} A\left(H_{i}\right)_{k} \\
H_{k} \downarrow \\
\operatorname{Mor}_{\mathcal{H}_{k}}(S, T) \stackrel{\cong}{\longrightarrow} \oplus_{i} \epsilon_{i} \downarrow \\
\bigoplus_{i} k,
\end{array}
$$

where $\epsilon_{i}: A\left(H_{i}\right)_{k} \rightarrow k$ is the augmentation map, the $A(G)_{k}$-module structure on the left hand side is given by any one of Lemma 2.22, and that of the right hand side is given through $\bigoplus_{i=1}^{n} \operatorname{Res}_{H_{i}}^{G}$.

In particular, $H_{k}: \operatorname{Mor}_{\mathcal{M}_{k}}(S, T) \rightarrow \operatorname{Mor}_{\mathcal{H}_{k}}(S, T)$ is always onto. 
Proof. The commutative diagram is obtained by

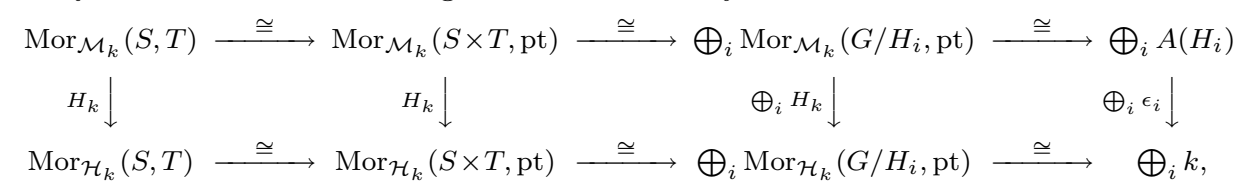

where the commutativities of the left, middle and the right squares follow from Lemma 2.6, Lemma 2.5, and Remark 2.15, respectively.

Remark 2.26. A different way of looking at the embedding of each factor $A\left(H_{i}\right) \rightarrow$ $\operatorname{Mor}_{\mathcal{M}_{k}}(S, T)$ in Proposition 2.25 is given as follows:

Fix each $G$-orbit $G /\left(H \cap K^{g}\right) \cong G \cdot\left(H \times g^{-1} K\right) \subset G / H \times G / K$. The corresponding factor $A\left(H \cap K^{g}\right)$ is embedded by the following map:

$$
\begin{aligned}
A\left(H \cap K^{g}\right) & \stackrel{\triangle_{\mathrm{pt}}}{\longrightarrow} \operatorname{Mor}_{\mathcal{M}_{k}\left(H \cap K^{g}\right)}(\mathrm{pt}, \mathrm{pt}) \stackrel{F_{H \cap K}^{G}}{\longrightarrow} \operatorname{Mor}_{\mathcal{M}_{k}(G)}\left(G /\left(H \cap K^{g}\right), G /\left(H \cap K^{g}\right)\right) \\
& \stackrel{c_{g} \circ \operatorname{Ind}_{H \cap K^{g}}^{K^{g}} \circ-\circ \operatorname{Res}_{H \cap K^{g}}^{H}}{\longrightarrow} \operatorname{Mor}_{\mathcal{M}_{k}(G)}(G / H, G / K),
\end{aligned}
$$

where $c_{g} \circ \operatorname{Ind}_{H \cap K^{g}}^{K^{g}} \circ-\circ \operatorname{Res}_{H \cap K^{g}}^{H}$ sends $f \in \operatorname{Mor}_{\mathcal{M}_{k}(G)}\left(G /\left(H \cap K^{g}\right), G /\left(H \cap K^{g}\right)\right)$ to $c_{g} \circ \operatorname{Ind}_{H \cap K^{g}}^{K^{g}} \circ f \circ \operatorname{Res}_{H \cap K^{g}}^{H}$.

Explicitly, the embedding is given by

$$
A\left(H \cap K^{g}\right) \hookrightarrow \operatorname{Mor}_{\mathcal{M}_{k}(G)}(G / H, G / K),
$$

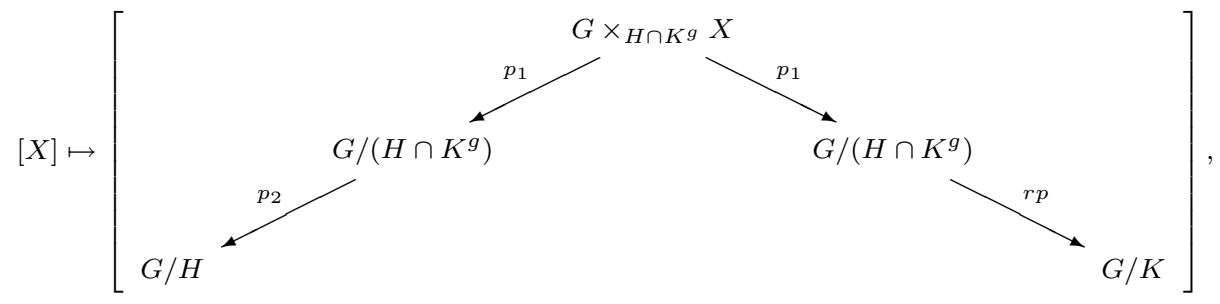

where $p_{1}$ and $p_{2}$ are obvious canonical projections and $r p: G /\left(H \cap K^{g}\right) \rightarrow G / K$ is the composite of the canonical projection onto $G / K^{g}$ and $r_{g^{-1}}: G / K^{g} \rightarrow$ $G / K, h K^{g}=h g^{-1} K g \mapsto h g^{-1} K$, the right multiplication by $g^{-1}$.

Corollary 2.27. If $H$ is a finite-index subgroup of $G$ and $S$ and $T$ are finite $G$-sets, suppose either $S=G \times_{H} S_{0}$ or $T=G \times_{H} T_{0}$ for some finite $H$-sets $S_{0}$ or $T_{0}$. Then the canonical $A(G)_{k}$-action on $\operatorname{Mor}_{\mathcal{M}_{k}(G)}(S, T)$ factors $\operatorname{Res}_{H}^{G}: A(G)_{k} \rightarrow A(H)_{k}$.

Definition 2.28. For any finite index subgroup $H$ of $G$ and a nonnegative integer $t \geq 0$, define a decreasing filtration $\left\{\mathcal{F}^{n} A(H)_{k}\right\}_{n=0}^{\infty}$ on $A(H)_{k}$ by

$$
\begin{aligned}
\mathcal{F}^{0} A(H)_{k} & =A(H)_{k}, \\
\mathcal{F}^{n} A(H)_{k} & =I(G)^{n} \cdot A(H)_{k},
\end{aligned}
$$

where $I(G)$ is the augmentation ideal of the Burnside ring $A(G)$. For any finite $G$-set $S=\coprod_{i} G / H_{i}$, define a decreasing filtration $\left\{\mathcal{F}^{n} A_{G}(S)_{k}\right\}_{n=0}^{\infty}$ on $A_{G}(S)_{k}$ by setting

$$
\mathcal{F}^{n} A_{G}(S)_{k}=\bigoplus_{i} \mathcal{F}^{n} A\left(H_{i}\right)_{k},
$$

where we have used the identification $A_{G}(S)_{k}=\bigoplus_{i} A\left(H_{i}\right)_{k}$ (Lemma 2.24).

Similarly, for any $G$-sets $S$ and $T$, we define a decreasing filtration

$$
\left\{\mathcal{F}^{n} \operatorname{Mor}_{\mathcal{M}_{k}}(S, T)\right\}_{n=0}^{\infty}
$$


using the filtration $\left\{\mathcal{F}^{n} A_{G}(S \times T)_{k}\right\}_{n=0}^{\infty}$ (see Lemma 2.24).

Definition 2.29. For any finite index subgroup $H$ of $G$ and a nonnegative integer $t \geq 0$, define a decreasing filtration $\left\{\mathcal{F}_{t}^{n} A(H)_{k}\right\}_{n=0}^{\infty}$ on $A(H)_{k}$ by induction on $t$ :

$$
\begin{aligned}
\mathcal{F}_{0}^{n} A(H)_{k}:= & I(H)_{k}^{n} \\
\mathcal{F}_{t}^{n} A(H)_{k}:= & \text { the ideal generated by elements of the form } \\
& \left(\operatorname{Ind}_{H_{1}}^{H} a_{1}\right) \cdot\left(\operatorname{Ind}_{H_{2}}^{H} a_{2}\right) \cdots\left(\operatorname{Ind}_{H_{l}}^{H} a_{l}\right),
\end{aligned}
$$

where $H_{i}$ is a finite index subgroup of $H$ and $a_{i} \in \mathcal{F}_{t-1}^{n_{i}} A\left(H_{i}\right)_{k}(1 \leq i \leq l)$ with $n_{1}+n_{2}+\cdots+n_{l} \geq n$.

Assembling these, we define a new filtration $\left\{\mathcal{F}_{\infty}{ }^{n} A(H)_{k}\right\}_{n=0}^{\infty}$ on $A(H)_{k}$ by

$$
\mathcal{F}_{\infty}{ }^{n} A(H)_{k}:=\bigcup_{t=0}^{\infty} \mathcal{F}_{t}^{n} A(H)_{k} .
$$

As special cases, we have

$$
\begin{gathered}
\mathcal{F}_{\infty}{ }^{0} A(H)_{k}=\mathcal{F}_{t}^{0} A(H)_{k}=\mathcal{F}_{0}^{0} A(H)_{k}=A(H)_{k}, \\
\mathcal{F}_{\infty}{ }^{1} A(H)_{k}=\mathcal{F}_{t}^{1} A(H)_{k}=\mathcal{F}_{0}^{1} A(H)_{k}=I(H)_{k} .
\end{gathered}
$$

Notice that this filtration $\left\{\mathcal{F}_{\infty}{ }^{n} A(H)_{k}\right\}_{n=0}^{\infty}$ on $A(H)_{k}$ does not depend upon any particular finite index embedding in $G$ at all, unlike the filtration $\left\{\mathcal{F}^{n} A(H)_{k}\right\}_{n=0}^{\infty}$.

For each finite $G$-set $S=\coprod_{i} G / H_{i}$, define a new decreasing filtration

$$
\left\{\mathcal{F}_{\infty}{ }^{n} A_{G}(S)_{k}\right\}_{n=0}^{\infty}
$$

on $A_{G}(S)_{k}$ by setting

$$
\mathcal{F}_{\infty}{ }^{n} A_{G}(S)_{k}=\bigoplus_{i} \mathcal{F}_{\infty}{ }^{n} A\left(H_{i}\right)_{k},
$$

where we have used the identification $A_{G}(S)_{k}=\bigoplus_{i} A\left(H_{i}\right)_{k}$ (Lemma 2.24).

Similarly, for each finite $G$-sets $S$ and $T$, we define a new decreasing filtration

$$
\left\{\mathcal{F}_{\infty}{ }^{n} \operatorname{Mor}_{\mathcal{M}_{k}}(S, T)\right\}_{n=0}^{\infty}
$$

using the filtration $\left\{\mathcal{F}_{\infty}{ }^{n} A_{G}(S \times T)_{k}\right\}_{n=0}^{\infty}$ (see Lemma 2.24).

Proposition 2.30. Suppose $G$ is a finite group. For any subgroup $H$ of $G$, define the completed Burnside rings $\left(A(H)_{k}\right)_{\mathcal{F}}$ and $\left(A(H)_{k}\right)_{\mathcal{F}_{\infty}}$ by

$$
\begin{aligned}
\left(A(H)_{k}\right)_{\mathcal{F}}^{\wedge} & :=\lim _{n} A(H)_{k} / \mathcal{F}^{n} A\left(H_{i}\right)_{k}, \\
\left(A(H)_{k}\right)_{\hat{\mathcal{F}}_{\infty}}: & :=\varliminf_{n} A(H)_{k} / \mathcal{F}_{\infty}{ }^{n} A\left(H_{i}\right)_{k} .
\end{aligned}
$$

Then the natural ring homomorphism $\left(A(H)_{k}\right)_{\mathcal{F}}^{\wedge} \rightarrow\left(A(H)_{k}\right)_{\mathcal{F}_{\infty}}$ is a topological isomorphism.

Proof. By definition, it is clear that

$$
\mathcal{F}^{n} A(H)_{k} \subseteq I(H)_{k}^{n} \subseteq \mathcal{F}_{\infty}{ }^{n} A(H)_{k} .
$$

Therefore, for any $n$ we must find an appropriate $N_{H}$ such that

$$
\mathcal{F}_{\infty}{ }^{N_{H}} A(H)_{k} \subseteq I(G)_{k}^{n} \cdot A(H)_{k}=\mathcal{F}^{n} A(H)_{k}
$$

to prove the claim. We prove this by induction on $H$ with respect to the inclusion.

First, (2.4) certainly holds for $H=\{e\}$ because $\mathcal{F}_{\infty}{ }^{n} A(\{e\})_{k}=\mathcal{F}^{n} A(\{e\})_{k}=0$ for any $n \geq 1$. 
Now assume (2.4) is proved for all maximal subgroups $H_{i}(i=1, \cdots, m)$ of $H$ with an appropriate integer $N_{H_{i}}$. Since the $I(G)$-adic topology and $I(H)$-adic topology determine the same topology on $A(H)$ for any subgroup $H$ of a finite group $G$ (see [18]), we may choose $M$ so that $I(H)_{k}^{M} \subseteq I(G)_{k}^{n} \cdot A(H)_{k}$. We claim that

$$
N_{H}:=M+\sum_{i=1}^{m}(M-1)\left(N_{H_{i}}-1\right)
$$

will do the job.

To see this, just notice that a general element in $\mathcal{F}_{\infty}{ }^{N_{H}} A(H)_{k}$ is a linear combination of elements of the form

$$
\left(\prod_{i=1}^{m}\left(\prod_{j=1}^{e_{i}}\left(\operatorname{Ind}_{H_{i}}^{H} a_{i, j}\right)\right)\right) \cdot a,
$$

where $e_{i} \geq 0, a_{i, j} \in \mathcal{F}_{\infty}{ }^{n_{i, j}} A\left(H_{i}\right)_{k}$ with $n_{i, j} \geq 1$, and $a \in I(H)_{k}^{e}$ for some $e \geq 0$ such that

$$
e+\sum_{i=1}^{m} \sum_{j=1}^{e_{i}} n_{i, j} \geq N_{H}=M+\sum_{i=1}^{m}(M-1)\left(N_{H_{i}}-1\right) .
$$

This is because any $\operatorname{Ind}_{K}^{H}$ from a finite index proper subgroup $K \subsetneq H$ factors through $\operatorname{Ind}_{H_{i}}^{H}$ for some finite-index maximal proper subgroup $H_{i}$ of $H$. Notice that a contradiction would occur if we falsely assume all of the following:

$$
\begin{aligned}
& e \leq M-1, \quad e_{i} \leq M-1(i=1, \cdots, m), \\
& n_{i, j} \leq N_{H_{i}}-1\left(i=1, \cdots, m, j=1, \cdots, e_{i}\right) .
\end{aligned}
$$

Thus, either 1) $e \geq M$, or 2) $e_{i} \geq M$ for some $i(i=1, \cdots, m)$, or 3$) n_{i, j} \geq N_{H_{i}}$ for some $i=1, \cdots, m$ and $j=1, \cdots, e_{i}$ occurs.

If 1) or 2) occurs, the element (2.5) clearly belongs to $I(H)_{k}^{M} \subseteq I(G)_{k}^{n} \cdot A(H)_{k}$.

If 3) occurs, by the inductive assumption, $a_{i, j} \in I(G)_{k}^{n} \cdot A\left(H_{i}\right)_{k}$, which implies $\operatorname{Ind}_{H_{i}}^{H} a_{i, j} \in I(G)_{k}^{n} \cdot A(H)_{k}$ because $\operatorname{Ind}_{H_{i}}^{H}$ is an $A(G)_{k}$-module homomorphism.

In this way, we have verified that the element (2.5) always belongs to $I(G)_{k}^{n}$. $A(H)_{k}$, which completes the proof of (2.4).

Lemma 2.31. For both filtrations in Definition 2.28 and Definition 2.29, the conjugation $c_{g}: A(H)_{k} \rightarrow A\left(H^{g}\right)_{k}$, the induction $\operatorname{Ind}_{K}^{H}: A(K)_{k} \rightarrow A(H)_{k}$, and the restriction $\operatorname{Res}_{K}^{H}: A(H)_{k} \rightarrow A(K)_{k}$ are filtration preserving.

Proof. $c_{g}$ : This is clear for the both filtrations.

$\operatorname{Ind}_{K}^{H}$ : The claim for $\left\{\mathcal{F}^{n}\right\}_{n=0}^{\infty}$ follows from the projection formula, which claims $\overline{\operatorname{Ind}_{K}^{H}}$ is an $A(H)_{k}$-module homomorphism (and so an $A(G)_{k}$-module homomorphism through $\left.\operatorname{Res}_{H}^{G}\right)$. The claim for $\left\{\mathcal{F}_{\infty}{ }^{n}\right\}_{n=0}^{\infty}$ follows from the definition; just notice that $\mathcal{F}_{\infty}{ }^{n} A(K)_{k}=\bigcup_{t=0}^{\infty} \mathcal{F}_{t}^{n} A(K)_{k}$ and

$$
\operatorname{Ind}_{K}^{H}\left(\mathcal{F}_{t}^{n} A(K)_{k}\right) \subseteq \mathcal{F}_{t+1}^{n} A(H)_{k} \subseteq \mathcal{F}_{\infty}{ }^{n} A(H)_{k} .
$$

$\operatorname{Res}_{K}^{H}$ : The claim for $\left\{\mathcal{F}^{n}\right\}_{n=0}^{\infty}$ follows from the fact that $\operatorname{Res}_{K}^{H}$ is a ring homo-

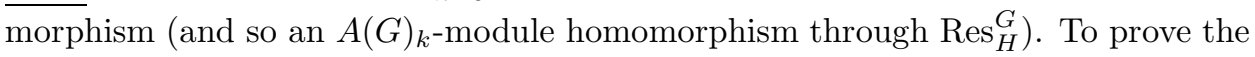
claim for $\left\{\mathcal{F}_{\infty}{ }^{n}\right\}_{n=0}^{\infty}$, we show that

$$
\operatorname{Res}_{K}^{H}\left(\mathcal{F}_{t}^{n} A(H)_{k}\right) \subseteq \mathcal{F}_{\infty}^{n} A(K)_{k}
$$


by induction on $t$. In fact, this is trivial for $t=0$, as $\mathcal{F}_{0}^{n} A(H)_{k}=I(H)_{k}^{n}$. Now assume this is proved for $t-1$. To show the claim for $t$, since $\operatorname{Res}_{K}^{H}$ is a ring homomorphism, it suffices to prove that

$$
\operatorname{Res}_{K}^{H} \operatorname{Ind}_{L}^{H}(a) \in \mathcal{F}_{\infty}^{m} A(K)_{k},
$$

where $L$ is a finite index subgroup of $H$ and $a \in \mathcal{F}_{t-1}^{m} A(L)_{k}$ (see Definition 2.29). However, the double coset formula claims it is a linear combination of elements of the form

$$
c_{g} \operatorname{Ind}_{K^{g^{-1}} \cap L}^{K^{\theta^{-1}}} \operatorname{Res}_{K^{g^{-1}} \cap L}^{L}(a),
$$

where $\operatorname{Res}_{K^{g^{-1}} \cap L}^{L}(a) \in \mathcal{F}_{\infty}{ }^{m} A\left(K^{g^{-1}} \cap L\right)_{k}$ by the inductive assumption, and Ind $_{K^{g^{-1}} \cap L}^{K^{-1}}$ and $c_{g}$ both preserve the filtration as was shown above. Thus the claim follows.

Lemma 2.32. For both filtrations in Definition 2.28 and Definition 2.29, the product $\mu: A(H)_{k} \otimes_{k} A(H)_{k} \rightarrow A(H)_{k}$ respects the filtration, i.e. it induces

$$
\begin{aligned}
\mathcal{F}^{a} A(H)_{k} \otimes \mathcal{F}^{b} A(H)_{k} & \rightarrow \mathcal{F}^{a+b} A(H)_{k} \\
\mathcal{F}_{\infty}{ }^{a} A(H)_{k} \otimes \mathcal{F}_{\infty}{ }^{b} A(H)_{k} & \rightarrow \mathcal{F}_{\infty}^{a+b} A(H)_{k}
\end{aligned}
$$

for any $a, b \geq 0$.

Proposition 2.33. For both filtrations in Definition 2.28 and Definition 2.29, we have the followings:

(i) All the isomorphisms in Lemma 2.24 are filtration preserving.

(ii) For any G-map $f: S \rightarrow T$ of finite $G$-sets, the induced map $f_{*}: A_{G}(S)_{k}$ $\rightarrow A_{G}(T)_{k}$ is filtration preserving.

(iii) The exterior pairing $\mu: A_{G}(S)_{k} \otimes A_{G}(T)_{k} \rightarrow A_{G}(S \times T)_{k}$ defined in Definition 2.19 respects the filtration, i.e. it induces

$$
\begin{aligned}
\mathcal{F}^{a} A_{G}(S)_{k} \otimes \mathcal{F}^{b} A_{G}(T)_{k} & \rightarrow \mathcal{F}^{a+b} A_{G}(S \times T)_{k}, \\
\mathcal{F}_{\infty}{ }^{a} A_{G}(S)_{k} \otimes \mathcal{F}_{\infty}{ }^{b} A_{G}(T)_{k} & \rightarrow \mathcal{F}_{\infty}{ }^{a+b} A_{G}(S \times T)_{k}
\end{aligned}
$$

for any $a, b \geq 0$.

Proof. (i) is easy to see. To show (ii), we simply notice that the map $f_{*}: A_{G}(S)_{k} \rightarrow$ $A_{G}(T)_{k}$, induced by a $G$-map of finite $G$-sets $f: S \rightarrow T$, is a linear combination of maps of the form $\operatorname{Ind}_{K}^{H}: A(K)_{k} \rightarrow A(H)_{k}$ with respect to the decomposition in Lemma 2.24. So the claim follows from Lemma 2.31.

To show (iii), we may assume both $S$ and $T$ are transitive $G$-sets, say $S=$ $G / H, T=G / K$ for some finite index subgroups $H$ and $K$. Choose a transitive orbit $G /\left(H \cap K^{g}\right) \cong G \cdot\left(H \times g^{-1} K\right) \subseteq G / H \times G / K$ and let $\pi: A_{G}(G / H \times G / H)_{k} \rightarrow$ $A_{G}\left(G / H \cap K^{g}\right)_{k}$ be the corresponding projection. Then, in view of Lemma 2.31 and Lemma 2.32, the claim will follow if we can show the composite

$$
\begin{aligned}
A(H)_{k} & \otimes_{k} A(K)_{k} \cong A_{G}(G / H)_{k} \otimes_{k} A_{G}(G / K)_{k} \\
& \stackrel{\mu}{\longrightarrow} A_{G}(G / H \times G / K)_{k} \stackrel{\pi}{\longrightarrow} A_{G}\left(G /\left(H \cap K^{g}\right)\right)_{k} \cong A\left(H \cap K^{g}\right)_{k}
\end{aligned}
$$

is the same as

$$
\begin{aligned}
& A(H)_{k} \otimes_{k} A(K)_{k} \stackrel{A(H)_{k} \otimes_{k} c_{g}}{\longrightarrow} A(H)_{k} \otimes_{k} A\left(K^{g}\right)_{k} \\
& \quad \stackrel{\operatorname{Res}_{H \cap K^{g}}^{H} \otimes_{k} \operatorname{Res}_{H \cap K^{g}}^{K^{g}}}{\longrightarrow} A\left(H \cap K^{g}\right)_{k} \otimes_{k} A\left(H \cap K^{g}\right)_{k} \stackrel{\mu}{\longrightarrow} A\left(H \cap K^{g}\right)_{k} .
\end{aligned}
$$


Now, pick a representative element $[X] \otimes[Y] \in A(H)_{k} \otimes_{k} A(K)_{k}$, where $X$ (resp. $Y$ ) is a finite $H$ set (resp. $K$ set). Then its image under the exterior pairing $\mu$ is

$$
\left[\left(G \times_{H} X\right) \times\left(G \times_{K} Y\right) \stackrel{p_{1} \times p_{2}}{\longrightarrow} G / H \times G / K\right] \in A_{G}(G / H \times G / K)_{k},
$$

where $p_{i}(i=1,2)$ are the canonical projection maps. To find out the $\pi$ image of this element, consider the following commutative diagram of $G$-maps between $G$-sets:

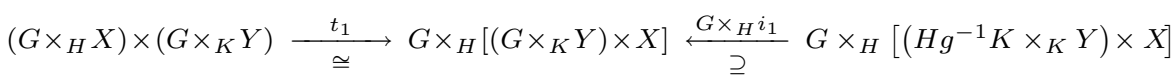

$$
\begin{aligned}
& p_{1} \times p_{2} \downarrow \quad G \times{ }_{H} p_{3} \downarrow-G \times{ }_{H} p_{4} \downarrow
\end{aligned}
$$

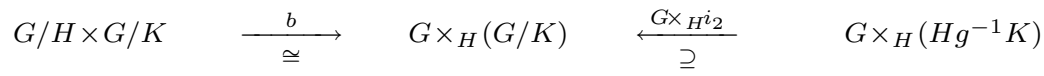

$$
\begin{aligned}
& \cong \downarrow \\
& G /\left(H \cap K^{g}\right),
\end{aligned}
$$

where $i_{1}$ and $i_{2}$ are canonical inclusions, $p_{1}$ and $p_{2}$ are canonical projections, and

$$
\begin{aligned}
& t_{1}:\left(\left(g_{1}, x\right),\left(g_{2}, y\right)\right) \mapsto\left(g_{1},\left(\left(g_{1}^{-1} g_{2}, y\right), x\right)\right) \text {, } \\
& b_{1}:\left(g_{1} H, g_{2} K\right) \mapsto\left(g_{1}, g_{1}^{-1} g_{2} K\right), \\
& p_{3}:((g, y), x) \quad \mapsto g K, \\
& p_{4}:\left(\left(h g^{-1} k, y\right), x\right) \mapsto h g^{-1} K .
\end{aligned}
$$

Notice that the desired $\pi$ image is exhibited in this commutative diagram as the right upper vertical map, which is the $G$-extension, i.e. the image under the isomorphism $A_{H}\left(H g^{-1} K\right)_{k} \cong A_{G}\left(G \times_{H}\left(H g^{-1} K\right)\right)_{k}$, of the left vertical map in the following commutative diagram of $H$-spaces and $H$-maps:

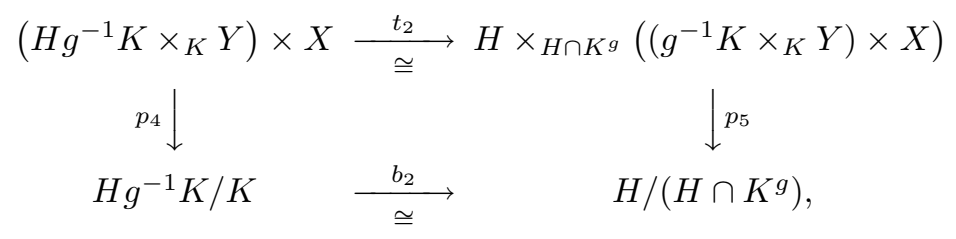

where $p_{5}$ is the canonical projection and

$$
\begin{aligned}
t_{2}:\left(\left(h g^{-1} k, y\right), x\right) & \mapsto\left(h,\left(g^{-1} k, y, h^{-1} x\right)\right), \\
b_{2}: h g^{-1} K & \mapsto h\left(H \cap K^{g}\right) .
\end{aligned}
$$

This latter commutative diagram exhibits the desired $\pi$ image as the $H$ extension, i.e. the image under the isomorphism $A\left(H \cap K^{g}\right)_{k} \cong A_{H}\left(H /\left(H \cap K^{g}\right)\right)_{k} \cong$ $A_{H}\left(H g^{-1} K\right)_{k}\left(\cong A_{G}\left(G \times_{H}\left(H g^{-1} K\right)\right)_{k}\right)$ of

$$
\left[\left(g^{-1} K \times_{K} Y\right) \times X\right] \in A\left(H \cap K^{g}\right)_{k} .
$$

However, $g^{-1} K \times_{K} Y$, regarded as a finite $K^{g}$-set (and so a finite $H \cap K^{g}$-set by restricting the action), represents the image of $[Y] \in A(K)_{k}$ under the conjugation $c_{g}: A(K)_{k} \rightarrow A\left(K^{g}\right)_{k}$ by Definition 2.16. This implies (2.6) is the same as (2.7), which completes the proof. 
Proposition 2.34. For both filtrations in Definition 2.28 and Definition 2.29, the composition

$$
\operatorname{Mor}_{\mathcal{M}_{k}}(S, T) \otimes \operatorname{Mor}_{\mathcal{M}_{k}}(T, U) \rightarrow \operatorname{Mor}_{\mathcal{M}_{k}}(S, U)
$$

respects the filtration, i.e. it induces

$$
\begin{aligned}
\mathcal{F}^{a} \operatorname{Mor}_{\mathcal{M}_{k}}(S, T) \otimes_{k} \mathcal{F}^{b} \operatorname{Mor}_{\mathcal{M}_{k}}(T, U) & \rightarrow \mathcal{F}^{a+b} \operatorname{Mor}_{\mathcal{M}_{k}}(S, U), \\
\mathcal{F}_{\infty}{ }^{a} \operatorname{Mor}_{\mathcal{M}_{k}}(S, T) \otimes_{k} \mathcal{F}_{\infty}{ }^{b} \operatorname{Mor}_{\mathcal{M}_{k}}(T, U) & \rightarrow \mathcal{F}_{\infty}{ }^{a+b} \operatorname{Mor}_{\mathcal{M}_{k}}(S, U)
\end{aligned}
$$

for any $a, b \geq 0$.

Proof. The claim for the filtration $\left\{\mathcal{F}^{n}\right\}_{n=0}^{\infty}$ is clear from Corollary 2.23.

For the filtration $\left\{\mathcal{F}_{\infty}{ }^{n}\right\}_{n=0}^{\infty}$ it suffices to check the claim when $T$ is a transitive $G$-set, say $G / H$. Then it is easy to check the commutativity of the following diagram:

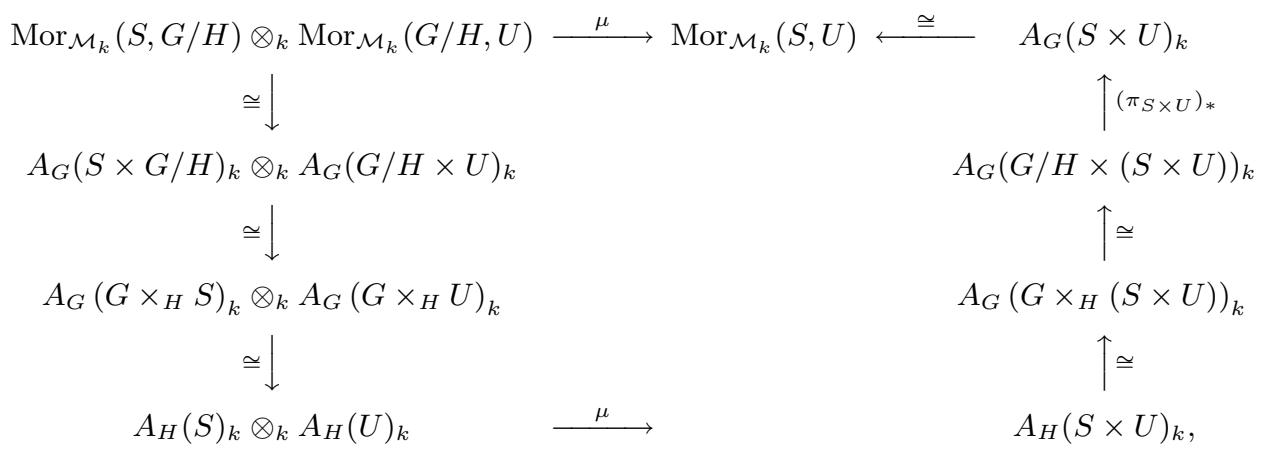

where $p_{S \times U}: G / H \times(S \times U) \rightarrow S \times U$ is the projection. In this diagram, the bottom $\mu$ respects the filtration as was shown in Proposition 2.33 (iii), and all the other maps are filtration preserving by Proposition 2.33 (i) (ii). Thus the claim follows.

Definition 2.35. For each $l \geq 1$, we define the reduced Mackey categories $\mathcal{M}_{k} / \mathcal{F}^{l}$ and $\mathcal{M}_{k} / \mathcal{F}_{\infty}{ }^{l}$ by

$$
\begin{aligned}
\operatorname{Ob}_{\mathcal{M}_{k} / \mathcal{F}^{l}} & =\operatorname{Ob}_{\mathcal{M}_{k} / \mathcal{F}_{\infty} l}=\{\text { finite } G \text {-sets }\} \\
\operatorname{Mor}_{\mathcal{M}_{k} / \mathcal{F} l}(S, T) & =\operatorname{Mor}_{\mathcal{M}_{k}}(S, T) / \mathcal{F}^{l} \operatorname{Mor}_{\mathcal{M}_{k}}(S, T), \\
\operatorname{Mor}_{\mathcal{M}_{k} / \mathcal{F}_{\infty} l}(S, T) & =\operatorname{Mor}_{\mathcal{M}_{k}}(S, T) / \mathcal{F}_{\infty}{ }^{l} \operatorname{Mor}_{\mathcal{M}_{k}}(S, T),
\end{aligned}
$$

where $S$ and $T$ are finite $G$-sets. Here the composition is induced by that of $\mathcal{M}_{k}$. Its well-definedness is guaranteed by Proposition 2.34.

Similarly, we define the completed Mackey categories $\left(\mathcal{M}_{k}\right)_{\mathcal{F}}^{\wedge}$ and $\left(\mathcal{M}_{k}\right)_{\mathcal{F}_{\infty}}$ by

$$
\begin{aligned}
& \mathrm{Ob}_{\left(\mathcal{M}_{k}\right) \hat{\mathcal{F}}}=\mathrm{Ob}_{\left(\mathcal{M}_{k}\right)_{\hat{\mathcal{F}}_{\infty}}}=\{\text { finite } G \text {-sets }\}, \\
& \operatorname{Mor}_{\left(\mathcal{M}_{k}\right) \hat{\mathcal{F}}}(S, T)=\varliminf_{l} \operatorname{Mor}_{\mathcal{M}_{k} / \mathcal{F}^{l}}(S, T) \text {, } \\
& \operatorname{Mor}_{\left(\mathcal{M}_{k}\right)_{\hat{\mathcal{F}}_{\infty}}}(S, T)=\varliminf_{l} \operatorname{Mor}_{\mathcal{M}_{k} / \mathcal{F}_{\infty} l}(S, T) \text {. }
\end{aligned}
$$


Notice that we have the following commutative diagram of categories and functors:

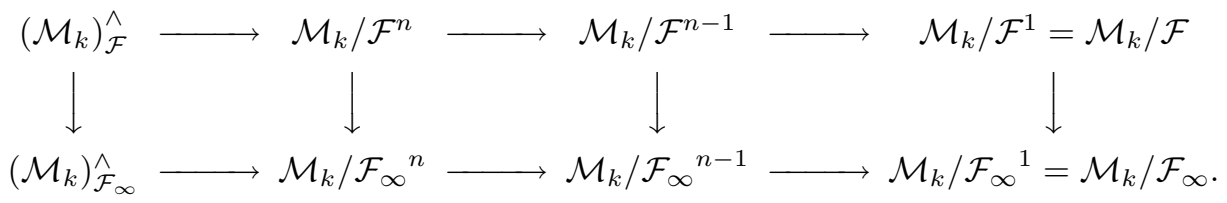

We call $\left(\mathcal{M}_{k}\right)_{\mathcal{F}}^{\wedge}$ the cohomotopical Mackey category over $k$. Note that

$$
\operatorname{Mor}_{\left(\mathcal{M}_{k}\right)_{\mathcal{\mathcal { F }}}}(S, T) \cong \operatorname{Mor}_{\mathcal{M}_{k}}(S, T) \otimes_{A(G)_{k}}\left(A(G)_{k}\right)_{I(G)_{k}}^{\wedge},
$$

when $G$ is a finite group.

Corollary 2.36. The Hurewicz functor $H_{k}: \mathcal{M}_{k} \rightarrow \mathcal{H}_{k}$ induces an isomorphism $\overline{H_{k}}: \mathcal{M}_{k} / \mathcal{F}_{\infty} \rightarrow \mathcal{H}_{k}$.

Proof. For any finite $G$-sets $S$ and $T$, we have the following commutative diagram from Proposition 2.25:

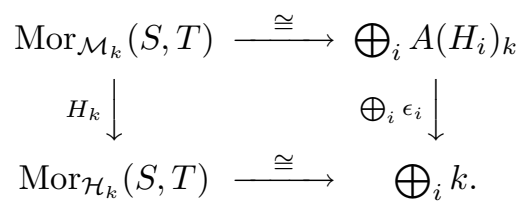

On the other hand, from Definition 2.35 and Definition 2.29, we have the following commutative diagram:

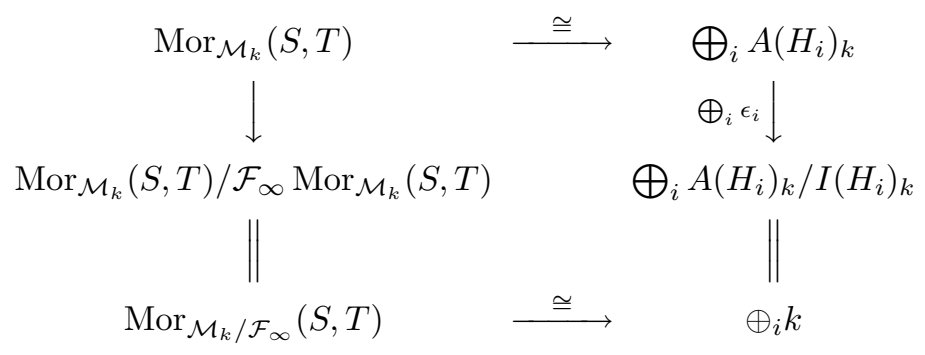

From these commutative diagrams, we immediately find that $H_{k}$ induces an isomorphism

$$
\operatorname{Mor}_{\mathcal{M}_{k} / \mathcal{F}_{\infty}}(S, T) \stackrel{\cong}{\rightrightarrows} \operatorname{Mor}_{\mathcal{H}_{k}}(S, T),
$$

which proves the claim.

Corollary 2.37. If $G$ is a finite group, then the natural map

$$
\left(\mathcal{M}_{k}\right)_{\mathcal{F}}^{\wedge} \rightarrow\left(\mathcal{M}_{k}\right)_{\mathcal{F}_{\infty}}^{\wedge}
$$

is a topological isomorphism.

Proof. This is an immediate consequence of Proposition 2.30, in view of Definition 2.28, Definition 2.29 and Definition 2.35.

Our attention is now focused upon the Hurewicz functor

$$
H_{k}:\left(\mathcal{M}_{k}\right)_{\mathcal{F}}^{\wedge} \rightarrow \mathcal{H}_{k}
$$

whose study is the main subject in $\S 4$. 


\section{Functors}

Having established basic properties of the underlying categories in the previous section, we now define various Mackey functors as $k$-additive functors from those categories.

As in the previous section, $G$ is a (possibly infinite) discrete group and $k$ is a commutative ring, unless otherwise stated.

Definition 3.1. We call a category $\mathcal{C}$ an additive category over $k$ if

1. For any objects $A$ and $B$ in $\mathcal{C}, \operatorname{Mor}_{\mathcal{C}}(A, B)$ is a $k$-module.

2. For any objects $A, B$, and $C$ in $\mathcal{C}$, the composition

$$
\operatorname{Mor}_{\mathcal{C}}(A, B) \otimes_{k} \operatorname{Mor}_{\mathcal{C}}(B, C) \rightarrow \operatorname{Mor}_{\mathcal{C}}(A, C)
$$

is $\mathrm{s} k$-module homomorphism.

A functor between additive categories over $k$ is called a $k$-additive functor, if the induced maps on the sets of morphisms are $k$-module homomorphisms.

Then we define a Mackey functor over $k$ as a $k$-additive (contravariant) functor from $\mathcal{M}_{k}$ to $\mathcal{C}$, an additive category over $k$. Since $\mathcal{M}_{k}=\mathcal{M}_{k}^{o p}$, there is no difference whether we choose covariant or contravariant in the definition of the Mackey functor. However, our namings of the maps in Definition 2.2 fit more naturally with the definition which requires contravariance.

Remark 3.2. The traditional definition of the Mackey functor [14, 10, 25, 22, 24] is essentially an additive functor from the Mackey category $\mathcal{M}_{k}$ to the category of $k$-modules, which transforms finite sum in $\mathcal{M}_{k}$ to finite sum in the category of $k$-modules, with the assumption that $G$ is finite. This latter condition is omitted in this paper. The use of $\mathcal{M}_{k}$ in the definition of the Mackey functor was first done by Linder [25] under a more general categorical setting.

Example 3.3. (i) Let $M$ be a $k G$-module. Then the correspondence $G / H \mapsto$ $H_{*}(H, M)$ defines a Mackey functor over $k$. Also, through the self-duality of the Mackey category, the correspondence $G / H \mapsto H^{*}(H, M)$ also defines a Mackey functor over $k$.

(ii) Let $X$ be a free $G$-space. Then the correspondence $S \mapsto \Sigma^{\infty} X_{+} \wedge_{G} S_{+}=$ $\Sigma^{\infty}\left(X \times_{G} S\right)_{+}$is a Mackey functor (over $\mathbb{Z}$ ) in the category of spectra.

More examples will be stated later.

Definition 3.4. Let $M$ be a Mackey functor over $k$ in the category $\mathcal{C}$. Then we call $\mathcal{M}_{M}$ the associated category of $M$ when

$$
\begin{aligned}
\operatorname{Ob}_{\mathcal{M}_{M}} & =\{\text { finite } G \text {-sets }\} \\
\operatorname{Mor}_{\mathcal{M}_{M}}(S, T) & =\operatorname{Im}\left(M: \operatorname{Mor}_{\mathcal{M}_{k}}(S, T) \rightarrow \operatorname{Mor}_{\mathcal{C}}(M(S), M(T))\right) .
\end{aligned}
$$

Definition 3.5. A cohomological Mackey functor over $k$ is a $k$-additive functor from $\mathcal{H}_{k}$.

Remark 3.6. (i) Originally $[14,1.4]$, the cohomological Mackey functor (over $k$ ) was defined to be a a Mackey functor $M$ (over $k$ ) in the sense of Remark 3.2, which further satisfies the following: If $H \subseteq K \subseteq G$ and $\beta \in M(G / K)$, then

$$
\operatorname{Ind}_{H}^{K} \circ \operatorname{Res}_{H}^{K}(\beta)=|K: H| \beta .
$$

The equivalence of these two conditions for those Mackey functors was originally proved by Yoshida [46]; we recover Yoshida's theorem below in Theorem 3.7. 
(ii) Of course, the homology and the cohomology of groups are the main examples of cohomological Mackey functors (see Example 3.3).

The following was originally proved by Yoshida [46] (for the case $\mathcal{C}$ is the category of $k$-modules) by a direct method:

Theorem 3.7. For Mackey functors in the category of $k$-modules, which transform finite sum in $\mathcal{M}_{k}$ to finite sum in the category of $k$-modules, the two definitions of the cohomological Mackey functors in Definition 3.5 and Remark 3.6 coincide.

Proof. Let $M: \mathcal{M}_{k} \rightarrow \mathcal{C}$ be a cohomological Mackey functor over $k$ in the sense of Remark 3.6. Then, by Proposition 2.25 and Remark 2.26, we can easily see that $M$, when restricted to each summand $A\left(H_{i}\right)_{k}$ in $\operatorname{Mor}_{\mathcal{M}_{k}}(S, T)$, factors through the augmentation $\epsilon_{i}: A\left(H_{i}\right) \rightarrow k$. This immediately implies (using the fact that $M$ transforms finite sum to finite sum) that $M$ factorizes $\mathcal{M}_{k} / \mathcal{F}_{\infty}$, which is isomorphic to the category of the finitely generated permutation $k G$-modules $\mathcal{H}_{k}$ by Corollary 2.36. The other implication is trivial.

We now come to the central concept of this paper.

Definition 3.8. We call $M$, a Mackey functor over $k$, a cohomotopical Mackey functor over $k$, if it is a $k$-additive functor from $\left(\mathcal{M}_{k}\right)_{\mathcal{F}}^{\wedge}$.

Example 3.9. (i) Any cohomological Mackey functor is cohomotopical.

(ii) Let $X$ be a $G$-equivariant infinite loop space (cf. [24]). Then the functor

$$
S \mapsto \pi_{n}\left(\operatorname{Map}_{G}(E G \times S, X)\right)^{\wedge}
$$

where the completion is given by the skeletal filtration of $E G$, is a cohomotopical Mackey functor. This functor may be viewed as the "cohomology of group with coefficient" in homotopical algebra [42], [38]. So we could call it as "cohomotopy of group with coefficient."

(iii) The Tate construction of [3] may be also viewed as a cohomotopical Mackey functor, just like (ii).

Now let us assume that $G$ is a finite group and $k=\mathbb{Z}_{p}^{\wedge}$. We would like to show a characterization of the cohomotopical Mackey functor for this particular case.

However, we must first define more notation: We write $A(G)_{p}^{\wedge}$ instead of $A(G) \otimes_{\mathbb{Z}}$ $\mathbb{Z}_{p}^{\wedge}$. Also, by $A(G)_{p+I(G)}^{\wedge}$, we mean the completion of $A(G)$ with respect to the ideal generated by $p$ and $I(G)$. Notice that this is also the same as $\left.(A(G) \wedge)_{p}^{\wedge}\right)_{I(G)_{p}^{\wedge}}$.

Fix a $p$-Sylow subgroup $G_{p}$ of $G$. Then we may write any finite $G$-set $S$ as

$$
S \cong \coprod_{i=1}^{n} G / H_{i}
$$

so that $H_{i} \cap G_{p}$ is a $p$-Sylow subgroup of $H_{i}$ for any $i=1,2, \cdots, n$. Set $\tilde{S}:=$ $\coprod_{i=1}^{n} G /\left(H_{i} \cap G_{p}\right)$ and define

$$
p_{S}:=\coprod_{i=1}^{n} p_{i}: \tilde{S} \cong \coprod_{i=1}^{n} G /\left(H_{i} \cap G_{p}\right) \rightarrow \coprod_{i=1}^{n} G / H_{i} \cong S
$$


where $p_{i}: G /\left(H_{i} \cap G_{p}\right) \rightarrow G / H_{i}$ is the canonical projection. We now set

$$
R_{S}:=\left[\begin{array}{ccc} 
& \tilde{S} & \\
p_{S} & 1_{\tilde{S}} \\
& & \tilde{S}
\end{array}\right] \in \operatorname{Mor}_{\mathcal{M}}(S, \tilde{S}) .
$$

Proposition 3.10. Let $G$ be a finite group and $k=\mathbb{Z}_{p}^{\wedge}$. The following four conditions about $M$, a Mackey functor over $\mathbb{Z}_{p}^{\wedge}$, are equivalent:

1. $M$ is a cohomotopical Mackey functor.

2. For any finite $G$-sets $T$ and $S$, the right composition with $R_{S}$ induces an injection

$$
M\left(R_{S} \circ-\right): \operatorname{Mor}_{\mathcal{C}}(M(T), M(S)) \rightarrow \operatorname{Mor}_{\mathcal{C}}(M(T), M(\tilde{S})) .
$$

3. The canonical action of $A(G)_{p}^{\wedge}$ on $\operatorname{Mor}_{\mathcal{C}}(M(T), M(S))$ factors through $A(G)_{p+I(G)}^{\wedge}$ for any finite $G$-sets $T$ and $S$.

4. The canonical action of $A(G)_{p}^{\wedge}$ on the set of the morphisms of the associated category of $M$ factors through $A(G)_{p+I(G)}^{\wedge}$.

Proof of $1 \Rightarrow 2$. It suffices to show that $R_{S} \in \operatorname{Mor}_{\left(\mathcal{M}_{k}\right)_{\mathcal{\mathcal { F }}}}(S, \tilde{S})$ is a split-injection, i.e. there exists $g \in \operatorname{Mor}_{\left(\mathcal{M}_{k}\right)_{\mathcal{F}}}(\tilde{S}, S)$ such that $g \circ R_{S}=1_{S} \in \operatorname{Mor}_{\left(\mathcal{M}_{k}\right)_{\hat{\mathcal{F}}}}(S, S)$. Now, from the definition of $R_{S}$, it suffices to prove the claim for a transitive finite $G$-set $S=G / H$. (Here $H$ is chosen so that $H \cap G_{P}$ is a $p$-Sylow subgroup of $H$ as before.) Set

$$
g_{S}:=\left[\begin{array}{ccc} 
& & \tilde{S} \\
& 1_{\tilde{S}} & p_{S} \\
\hdashline & & S
\end{array}\right] \in \operatorname{Mor}_{\left(\mathcal{M}_{k}\right)_{\mathcal{F}}}(\tilde{S}, S) .
$$

Then, when $S=G / H$, the composition $g_{S} \circ R_{S} \in \operatorname{Mor}_{\left(\mathcal{M}_{k}\right)_{\mathcal{F}}}(G / H, G / H)$ is given by the image of $\left[H /\left(H \cap G_{p}\right)\right]$ under the composite (see Lemma 2.21)):

$$
A(H)_{p+I(H)}^{\wedge} \stackrel{\triangle_{\mathrm{pt}}}{\longrightarrow} \operatorname{Mor}_{\left(\mathcal{M}_{k}\right)_{\mathcal{F}}(H)}(\mathrm{pt}, \mathrm{pt}) \rightarrow \operatorname{Mor}_{\left(\mathcal{M}_{k}\right)_{\hat{\mathcal{F}}}(G)}(G / H, G / H) .
$$

However, $\left[H /\left(H \cap G_{p}\right)\right] \in A(H)_{p+I(H)}^{\wedge}$ is a unit, which implies

$$
g_{S} \circ R_{S} \in \operatorname{Mor}_{\left(\mathcal{M}_{k}\right)_{\mathcal{F}}}(G / H, G / H)
$$

is also a unit. Now the claim follows immediately.

Proof of $2 \Rightarrow 3$. Consider the following commutative diagram of the ( $p$-completed) Burnside ring actions:

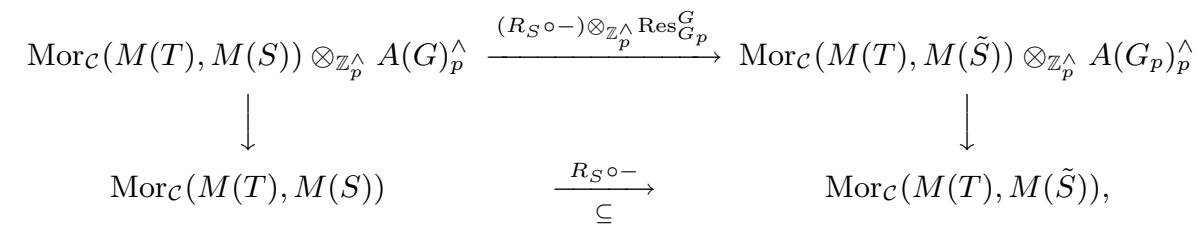

where the commutativity follows from Corollary 2.27 and the bottom map is injective by the assumption 1 . 
Therefore $A(G)_{p}^{\wedge}$-action on $\operatorname{Mor}_{\mathcal{C}}(M(T), M(S))$ factors through $\operatorname{Im}\left(A(G)_{p}^{\wedge} \rightarrow\right.$ $\left.A\left(G_{p}\right)_{p}^{\wedge}\right)$, which can be canonically identified with $A(G)_{p+I(G)}^{\wedge}$ from our knowledge of the $I(G)$-adic topology and $p$-adic topology of $A(G)$ (see $[18,31]$ ).

Proof of $3 \Rightarrow 4$. This is immediate from the definition of the associated category (see Definition 3.4).

Proof of $4 \Rightarrow 1$. This follows from $A(G)_{p+I(G)}^{\wedge}=\left(A(G)_{k}\right)_{I(G)_{k}}^{\wedge}$ with $k=\mathbb{Z}_{p}^{\wedge}$, since $\operatorname{Mor}_{\left(\mathcal{M}_{k}\right)_{\mathcal{\mathcal { F }}}}(S, T)=\operatorname{Mor} \mathcal{M}_{k}(S, T) \otimes_{A(G)_{k}}\left(A(G)_{k}\right)_{I(G)_{k}}^{\wedge}$ when $G$ is a finite group.

Corollary 3.11. Let $X$ be a free $G$-space. Then the correspondence (cf. Example 3.3)

$$
S \mapsto\left(\Sigma^{\infty} X \times_{G} S_{+}\right)_{p}^{\wedge}
$$

is a cohomotopical Mackey Mackey functor over $\mathbb{Z}_{p}^{\wedge}$.

Remark 3.12. (i) Proposition 3.10 and its proof give yet another characterization of cohomotopical Mackey functors over $\mathbb{Z}_{p}^{\wedge}$ when $G$ is finite. Let $\left(A_{G}\right)_{p+I(G)}^{\wedge}$ be the Green functor $S \mapsto A_{G}(S)_{p+I(G)}^{\wedge}$, where $A_{G}$ is as in Definition 2.18 (which is the (Burnside) Green functor defined and denoted by $\Omega$ in [11, p.303]), and the completion is with respect to the ideal $p+I(G) \subseteq A(G)$ through its $A(G)$ module structure. Then the cohomotopical Mackey functors over $\mathbb{Z}_{p}^{\wedge}$ for $G$ finite are nothing but Green modules over the $G / G_{p}$-injective Green functor $\left(A_{G}\right)_{p+I(G)}^{\wedge}$. See Propositions 1.2 and $1.1^{\prime}$ of [11] for some consequences.

(ii) Proposition 3.102 implies that a $p$-Sylow subgroup is a detecting subgroup.

\section{The MAIN THEOREM AND ITS PROOFS}

In this section, we state and prove our main results. First we prove a list of results concerning the category $\left(\mathcal{M}_{k}\right)_{\mathcal{F}_{\infty}}^{\wedge}$ and its relations with the Hecke category $\mathcal{H}_{k}$ through the Hurewicz functor.

Proposition 4.1. 1. For any finite $G$-set $S$, the Hurewicz functor $H_{k}:\left(\mathcal{M}_{k}\right)_{\mathcal{F}_{\infty}}^{\wedge}$ $\rightarrow \mathcal{H}_{k}$ induces a surjection of $k$-algebras

$$
H_{k}:\left(\operatorname{Mor}_{\left(\mathcal{M}_{k}\right)_{\mathcal{F}_{\infty}}}(S, S)\right) \rightarrow\left(\operatorname{Mor}_{\mathcal{H}_{k}}(S, S)\right)
$$

with its kernel quasi-regular, i.e. for any $k \in \operatorname{Ker}\left(H_{k}:\left(\mathcal{M}_{k}\right)_{\mathcal{F}_{\infty}} \rightarrow \mathcal{H}_{k}\right)$, $1+k \in \operatorname{Mor}_{\left(\mathcal{M}_{k}\right)_{\hat{\mathcal{F}}_{\infty}}}(S, S)$ is a unit.

2. For any finite $G$-set $S$, the Hurewicz functor $H_{k}:\left(\mathcal{M}_{k}\right)_{\mathcal{F}_{\infty}}^{\wedge} \rightarrow \mathcal{H}_{k}$ induces a surjection of the group of units

$$
H_{k}^{\times}:\left(\operatorname{Mor}_{\left(\mathcal{M}_{k}\right)_{\mathcal{F}_{\infty}}}(S, S)\right)^{\times} \rightarrow\left(\operatorname{Mor}_{\mathcal{H}_{k}}(S, S)\right)^{\times} .
$$

3. The Hurewicz functor induces an isomorphism of semi-simple rings:

$$
\begin{aligned}
& \left(\operatorname{Mor}_{\left(\mathcal{M}_{k}\right)_{\mathcal{F}_{\infty}}}(S, S)\right) / J\left(\operatorname{Mor}_{\left(\mathcal{M}_{k}\right)_{\mathcal{F}_{\infty}}}(S, S)\right) \\
& \quad \stackrel{\simeq}{\longrightarrow}\left(\operatorname{Mor}_{\mathcal{H}_{k}}(S, S)\right) / J\left(\operatorname{Mor}_{\mathcal{H}_{k}}(S, S)\right) \\
& =\left(\operatorname{Hom}_{k G}(k S, k S)\right) / J\left(\operatorname{Hom}_{k G}(k S, k S)\right) .
\end{aligned}
$$


Here $J(?)$ is the Jacobson radical of (?), i.e. the intersection of all maximal left ideals (cf. $[4,9])$.

4. Assume further that $G$ is finite and $k=\mathbb{Z}_{p}^{\wedge}$. Then all of the above 1, 2, and 3 are also valid when $\left(\mathcal{M}_{k}\right)_{\mathcal{F}_{\infty}}^{\wedge}$ is replaced with $\left(\mathcal{M}_{k}\right)_{\mathcal{F}}^{\wedge}$. Furthermore, there exists $n_{0}>0$ such that, for any $f_{i} \in \operatorname{Ker}\left(H_{p}: \operatorname{Mor}_{\left(\mathcal{M}_{k}\right)_{\hat{\mathcal{F}}_{\infty}}}\left(S_{i}, S_{i+1}\right) \rightarrow\right.$ $\left.\operatorname{Mor}_{\mathcal{H}_{k}}\left(S_{i}, S_{i+1}\right)\right)(i=1, \cdots, n)$ with $n \geq n_{0}$, we have

$$
f_{n} \circ f_{n-1} \circ \cdots \circ f_{2} \circ f_{1} \in p \operatorname{Mor}_{\left(\mathcal{M}_{k}\right)_{\hat{\mathcal{F}}_{\infty}}}\left(S_{1}, S_{n+1}\right) .
$$

Proof of 1, 2 and 3. For 1, it is certainly surjective by Proposition 2.25. The remaining claim of 1 follows immediately from Proposition 2.34, which allows us to construct its inverse $\sum_{n=0}^{\infty}(-1)^{n} k^{n}$ explicitly. Then 2 immediately follows from 1 . Furthermore, 3 is also an immediate consequence of 1 [4, 9.15,15.3].

Proof of 4. The first claim is an immediate consequence of 1, 2, 3 and Corollary 2.37 .

For the second claim, from Proposition 2.34, Corollary 2.37, and Proposition 2.25, it suffices to show there exists a certain $n_{0}$ such that for any subgroup $H$ of a finite group $G$

$$
\left(I(G)_{\mathbb{Z}_{p}^{\wedge}}\right)^{n} \cdot A(H)_{p+I(H)}^{\wedge} \subseteq p A(H)_{p+I(H)}^{\wedge}
$$

for any $n \geq n_{0}$, becausee $A(H)_{p+I(H)}^{\wedge}=\left(A(H)_{\mathbb{Z}_{\hat{p}}}\right)_{I(G)_{\mathbb{Z}_{\hat{p}}}}^{\wedge}$.

To show (4.1), we choose a $p$-subgroup $H_{p}$ of $H$, and notice that

$$
\operatorname{Res}_{H_{p}}^{H}: A(H)_{p+I(H)}^{\wedge} \longmapsto A\left(H_{p}\right)_{p+I\left(H_{p}\right)}^{\wedge}
$$

is a topological split injection (cf. [31]) and the topology on $A\left(H_{p}\right)_{p+I\left(H_{p}\right)}$ is given by the $p$-adic topology since $H_{p}$ is a $p$-group ([18]).

Therefore, the $I(G)_{\mathbb{Z}_{\hat{p}}}$-adic topology on $A(H)_{p+I(H)}^{\wedge}$ also becomes a $p$-adic topology, and so (since there are just finitely many subgroups inside a finite group $G$ ), there certainly exists some $n_{0}$ which satisfies (4.1).

Notice that, for $G$ finite and $k=\mathbb{Z}_{p}^{\wedge}, 4$ implies 1,2 and 3 . We now offer a topological proof of this particular case, which is a simplified version of our original proof in [31]. Although the proof requires the Segal conjecture, we hope this would give the reader better ideas about the underlying relationship between algebra and topology.

A topological proof of 4. By Carlsson's affirmative solution of the Segal conjecture [6], the composite

$$
\begin{aligned}
& \alpha_{\left(S_{1}, S_{n+1}\right)}: \operatorname{Mor}_{\left(\mathcal{M}_{k}\right)_{\mathcal{\mathcal { F }}}}\left(S_{1}, S_{n+1}\right) \cong \operatorname{Mor}_{\left(\mathcal{M}_{k}\right)_{\mathcal{F}}}\left(S_{1} \times S_{n+1}, \mathrm{pt}\right) \\
& \cong\left(\left\{\left(S_{1} \times S_{n+1}\right)_{+}, S^{0}\right\}_{G}\right)_{p+I(G)}^{\wedge} \cong\left\{E G_{+} \wedge_{G}\left(S_{1} \times S_{n+1}\right)_{+}, S^{0}\right\}_{p}^{\wedge}
\end{aligned}
$$

is an isomorphism. Under this isomorphism, the composite $f_{n} \circ f_{n-1} \circ \cdots \circ f_{2} \circ f_{1}$ is sent to

$$
\begin{aligned}
E G_{+} \wedge_{G}\left(S_{1} \times S_{n+1}\right)_{+} & \stackrel{1 \wedge\left(f_{1} \times 1\right)}{\longrightarrow} E G_{+} \wedge_{G}\left(S_{2} \times S_{n+1}\right)_{+} \stackrel{1 \wedge\left(f_{2} \times 1\right)}{\longrightarrow} \cdots \\
\ldots & \stackrel{1 \wedge\left(f_{n} \times 1\right)}{\longrightarrow} E G_{+} \wedge_{G}\left(S_{n+1} \times S_{n+1}\right)_{+} \stackrel{\alpha_{\left(S_{n+1}, S_{n+1}\right)}\left(1_{S_{n+1}}\right)}{\longrightarrow} S_{+}^{0} .
\end{aligned}
$$


Now the point is that the correspondence

$$
S \rightsquigarrow H_{*}\left(E G_{+} \wedge_{G}\left(S \times S_{n+1}\right)_{+} ; \mathbb{Z} / p \mathbb{Z}\right)
$$

defines a cohomological Mackey functor, which, together with the assumption, implies

$$
\left(1 \wedge\left(f_{i} \wedge 1\right)\right)_{*}=0 \quad(i=1, \cdots n) .
$$

Therefore, $\alpha_{\left(S_{1}, S_{n+1}\right)}\left(f_{n} \circ f_{n-1} \circ \cdots \circ f_{2} \circ f_{1}\right)$ has Adams filtration at least $n$. However, Carlsson's solution [6] of the Segal conjecture implies that

$$
\left\{E G_{+} \wedge_{G}\left(S_{1} \times S_{n+1}\right)_{+}, S^{0}\right\}_{p}^{\wedge}
$$

is a finitely generated free $\mathbb{Z}_{p}^{\wedge}$-module. Therefore, there exists some $n_{0}$ such that any element with the Adams filtration $\geq n_{0}$ is a multiple of $p$. Now the claim follows immediately.

Remark 4.2. 4.1 can be regarded as a representation theoretical analogue of the Nishida nilpotency theorem [36]. This states that any element in the kernel of the stable Hurewicz homomorphism $H: \pi_{*}^{s}\left(S^{0}\right) \rightarrow H_{*}\left(S^{0}\right)$ is nilpotent.

We have now come to the main theorem of this paper.

Main Theorem. (i) Let $G$ be a (possibly infinite) discrete group, and let $S$ and $T$ be finite $G$-sets such that the corresponding permutation $k G$-modules are isomorphic:

$$
k S \cong k T, \text { as } k G \text {-modules } .
$$

Then for any cohomotopical Mackey functor $M$,

$$
M(S) \cong M(T) \text {. }
$$

(ii) Suppose $k=\mathbb{Z}_{p}^{\wedge}$. Then for any finite $G$-set $S$ and any idempotent $e \in$ $\operatorname{Hom}_{k G}(k S, k S)=\operatorname{Mor}_{\mathcal{H}_{k}}(S, S)$, there exists an idempotent $\tilde{e} \in \operatorname{Mor}_{\mathcal{M}_{k}}(S, S)$, which lifts e.

Proof of (i). From the assumption, there are

$$
f \in \operatorname{Hom}_{k G}(k S, k T)=\operatorname{Mor}_{\mathcal{H}_{k}(G)}(S, T)
$$

and

$$
g \in \operatorname{Hom}_{k G}(k T, k S)=\operatorname{Mor}_{\mathcal{H}_{k}(G)}(T, S)
$$

such that

$$
f \circ g=1_{k T}, \quad g \circ f=1_{k S} .
$$

Now, choose a finite index normal subgroup $N_{0}$ which is contained in the intersection of all the isotropy subgroups of $S$ and $T$, as in Proposition 2.9. Then from the following commutative diagrams (see Proposition 2.9) and Proposition 2.25:

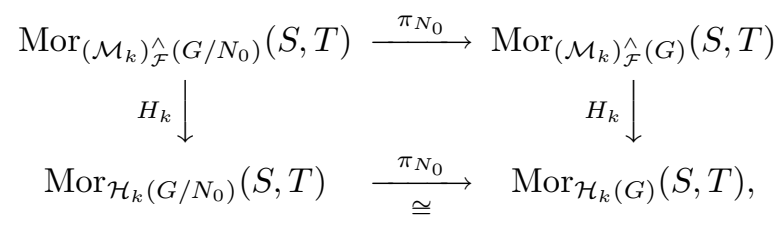




$$
\begin{array}{ccc}
\operatorname{Mor}_{\left(\mathcal{M}_{k}\right) \hat{\mathcal{F}}\left(G / N_{0}\right)}(T, S) \stackrel{\pi_{N_{0}}}{\longrightarrow} \operatorname{Mor}_{\left(\mathcal{M}_{k}\right) \hat{\mathcal{F}}(G)}(T, S) \\
H_{k} \downarrow & H_{k} \downarrow \\
\operatorname{Mor}_{\mathcal{H}_{k}\left(G / N_{0}\right)}(T, S) & \stackrel{\pi_{N_{0}}}{\longrightarrow} & \operatorname{Mor}_{\mathcal{H}_{k}(G)}(T, S),
\end{array}
$$

we may find some lifts $\tilde{f} \in \operatorname{Mor}_{\left(\mathcal{M}_{k}\right)_{\mathcal{F}}(G)}(S, T), \quad \bar{f} \in \operatorname{Mor}_{\left(\mathcal{M}_{k}\right)_{\mathcal{F}}\left(G / N_{0}\right)}(S, T), \quad \tilde{g} \in$ $\operatorname{Mor}_{\left(\mathcal{M}_{k}\right)_{\mathcal{F}}(G)}(T, S)$, and $\bar{g} \in \operatorname{Mor}_{\left(\mathcal{M}_{k}\right)_{\mathcal{F}}\left(G / N_{0}\right)}(T, S)$, so that

$$
\pi_{N_{0}}(\bar{f})=\tilde{f}, H_{k}(\tilde{f})=f, \pi_{N_{0}}(\bar{g})=\tilde{g}, H_{k}(\tilde{g})=g .
$$

Although

$$
\begin{aligned}
& \bar{f} \circ \bar{g} \neq 1 \in \operatorname{Mor}_{\left(\mathcal{M}_{k}\right)_{\mathcal{F}}\left(G / N_{0}\right)}(T, T), \\
& \bar{g} \circ \bar{f} \neq 1 \in \operatorname{Mor}_{\left(\mathcal{M}_{k}\right)_{\mathcal{\mathcal { F }}}\left(G / N_{0}\right)}(S, S)
\end{aligned}
$$

in general, their errors respectively belong to

$$
\begin{aligned}
& \operatorname{Ker}\left(H_{k}: \operatorname{Mor}_{\left(\mathcal{M}_{k}\right)_{\mathcal{F}}\left(G / N_{0}\right)}(T, T) \rightarrow \operatorname{Mor}_{\mathcal{H}_{k}}(T, T)\right), \\
& \operatorname{Ker}\left(H_{k}: \operatorname{Mor}_{\left(\mathcal{M}_{k}\right)_{\mathcal{F}}\left(G / N_{0}\right)}(S, S) \rightarrow \operatorname{Mor}_{\mathcal{H}_{k}}(S, S)\right) .
\end{aligned}
$$

Thus, by Proposition 4.1 (which we may apply as $G / N$ is finite), both $\bar{f} \circ \bar{g}$ and $\bar{g} \circ \bar{f}$ are units in the $k$-algebras $\operatorname{Mor}_{\left(\mathcal{M}_{k}\right)_{\mathcal{F}}\left(G / N_{0}\right)}(T, T)$ and $\operatorname{Mor}_{\left(\mathcal{M}_{k}\right)_{\mathcal{F}}\left(G / N_{0}\right)}(S, S)$, respectively.

Since

$$
\begin{aligned}
& \operatorname{Mor}_{\left(\mathcal{M}_{k}\right)_{\hat{\mathcal{F}}}\left(G / N_{0}\right)}(T, T) \stackrel{\pi_{N_{0}}}{\longrightarrow} \operatorname{Mor}_{\left(\mathcal{M}_{k}\right)_{\hat{\mathcal{F}}}(G)}(T, T), \\
& \operatorname{Mor}_{\left(\mathcal{M}_{k}\right)_{\hat{\mathcal{F}}}\left(G / N_{0}\right)}(S, S) \stackrel{\pi_{N_{0}}}{\longrightarrow} \operatorname{Mor}_{\left(\mathcal{M}_{k}\right)_{\hat{\mathcal{F}}}(G)}(S, S)
\end{aligned}
$$

are $k$-algebra homomorphisms, we now know that both $\tilde{f} \circ \tilde{g}$ and $\tilde{g} \circ \tilde{f}$ are units in the $k$-algebras $\operatorname{Mor}_{\left(\mathcal{M}_{k}\right)_{\mathcal{F}}(G)}(T, T)$ and $\operatorname{Mor}_{\left(\mathcal{M}_{k}\right)_{\mathcal{F}}(G)}(S, S)$, respectively. This implies the objects $S$ and $T$ are equivalent in the category $\left(\mathcal{M}_{k}\right)_{\mathcal{F}}^{\wedge}$. Now the claim follows.

Proof of (ii). Arguing as the proof of (i), we may assume $G$ is finite. Now let $e^{\prime} \in \operatorname{Mor}_{\left(\mathcal{M}_{k}\right)_{\mathcal{\mathcal { F }}}}(S, S)$ be any lift of $e$. Since $e$ is an idempotent, we may write

$$
e^{\prime 2}=e^{\prime}+l
$$

where $l \in \operatorname{Ker}\left(H_{p}: \operatorname{Mor}_{\left(\mathcal{M}_{k}\right)_{\mathcal{F}}}(S, S) \rightarrow \operatorname{Mor}_{\mathcal{H}_{k}}(S, S)\right)$. Then, from Proposition 4.1, there exists some $n_{1} \geq 1$ such that $k^{p^{n_{1}}}=p x$ for some $x \in \operatorname{Mor}_{\left(\mathcal{M}_{k}\right)_{\mathcal{\mathcal { F }}}}(S, S)$. Set $e^{\prime \prime}:=e^{p^{n_{1}}}$. Then $e^{\prime \prime}$ is another lift of $e$ such that

$$
e^{\prime \prime 2}=e^{\prime \prime}+p y
$$

for some $y \in \operatorname{Mor}_{\left(\mathcal{M}_{k}\right)_{\mathcal{F}}}(S, S)$. Since $\operatorname{Mor}_{\left(\mathcal{M}_{k}\right)_{\mathcal{\mathcal { F }}}}(S, S)$ is $p$-adically complete (this is because $G$ is finite), we can construct an idempotent lift of $e$ by the standard argument using $e^{\prime \prime}$; just check that $\left\{e^{\prime \prime p^{n}}\right\}_{n=1}^{\infty}$ is a Cauchy sequence with respect to the $p$-adic topology and let $\tilde{e} \in \operatorname{Mor}_{\left(\mathcal{M}_{k}\right)_{\mathcal{\mathcal { F }}}}(S, S)$ be the limit. Then $\tilde{e}$ is easily seen to be an idempotent lift of $e$. 


\section{WEBB'S FORMULAE AND APPLICATIONS}

In [44], Webb proved interesting combinatorial formulae for Tate and ordinary cohomology of finite groups. Since the essence of his results is the corresponding combinatorial formulae about permutation $\mathbb{Z}_{p}^{\wedge} G$-modules, we may use it as an input of our Main Theorem. For the rest of this paper, we assume $G$ is a finite group. We first recall Webb's results:

Theorem 5.1 (Theorem $\mathrm{A}^{\prime}$ of [44]). Let $G$ act simplicially on the simplicial complex $\triangle$, suppose for each simplex $\sigma$ the isotropy group $G_{\sigma}$ fixes $\sigma$ pointwise, and let $p$ be a fixed prime. Assume that one of the following conditions holds:

1. for each $H \in \mathcal{C}$ with $O_{p}(H) \neq 1$ the fixed point complex $\triangle^{H}$ has Euler characteristic $\chi\left(\triangle^{H}\right)=1$,

2. for each cyclic subgroup $H$ of order $p, \triangle^{H}$ is acyclic.

Then we have an isomorphism

$$
\mathbb{Z}_{p}^{\wedge} G / G \equiv \sum_{\sigma \in \triangle / G}(-1)^{\operatorname{dim}(\sigma)} \mathbb{Z}_{p}^{\wedge} G / G_{\sigma},
$$

as $\mathbb{Z}_{p}^{\wedge} G$-modules, modulo projectives of the form $\mathbb{Z}_{p}^{\wedge} G / H$ with $(p,|H|)=1$.

As was mentioned in [44], Quillen [39] provided interesting examples for this theorem.

Theorem 5.2 ([39]). Theorem $A^{\prime}$ is valid when $\triangle=\mathcal{A}$ is the poset of all nonidentity elementary abelian p-subgroups of $G$, or when $\mathcal{P}$ is the poset of non-identity p-subgroups of $G$, or the Tits building of a finite Chevalley group in defining characteristic $p$.

In a step of his proof in [44], Webb proved the following result, which doesn't involve any equivalence modulo projectives. It is based upon Conlon's deep result [8] on the detecting subgroups of the Green ring, and the idempotent formula of the Burnside ring by $[13,47]$. We denote the class of mod- $p$ cyclic subgroups of $G$ by $\mathcal{C}$ :

$$
\begin{array}{r}
\mathcal{C}=\left(H \subseteq G \mid O_{p}(H), p \text {-Sylow of } H, \text { is a normal subgroup of } G\right. \\
\text { and } \left.H / O_{p}(H) \text { is cyclic }\right) .
\end{array}
$$

Theorem 5.3 (Theorem $\mathrm{D}^{\prime}$ of [44]). Let $\mathcal{X}$ be a class of subgroups of $G$ which is closed under taking conjugates and forming subgroups, and with $\mathcal{X} \supseteq \mathcal{C}$. Then in the Green ring of $G$ defined over $\mathbb{Q}$,

$$
\mathbb{Z}_{p}^{\wedge} G / G=\sum_{H \in \mathcal{X}} \frac{\mu(H)}{[G: H]} \mathbb{Z}_{p}^{\wedge} G / H,
$$

where $\mu: \mathcal{X} \rightarrow \mathbb{Z}$ is the function defined by the equations

$$
\sum_{J \subseteq K \in \mathcal{X}} \mu(K)=1 \text { for every } J \in \mathcal{X} .
$$

To apply Theorem 5.1 and Theorem 5.2, we provide the following lemma.

Lemma 5.4. Let $M$ be a cohomotopical Mackey functor over $\mathbb{Z}_{p}^{\wedge}$ such that $M(G /\{e\})=0$. Then for any subgroup $H \subseteq G$ such that $(|H|: p)=1$ and $M(G / H)=0$. 
Proof. Since $\mathbb{Z}_{p}^{\wedge} G / H$ is a projective $\mathbb{Z}_{p} G$-module, there exists some $n \in \mathbb{N}$ and

$$
f \in \operatorname{Hom}_{\mathbb{Z}_{p}^{\wedge} G}\left(\mathbb{Z}_{p}^{\wedge} G / H,\left(\mathbb{Z}_{p}^{\wedge} G\right)^{\oplus n}\right), g \in \operatorname{Hom}_{\mathbb{Z}_{p}^{\wedge}}\left(\left(\mathbb{Z}_{p}^{\wedge} G\right)^{\oplus n}, \mathbb{Z}_{p}^{\wedge} G / H\right)
$$

such that

$$
g \circ f=1_{\mathbb{Z}_{p}^{\wedge} G / H} \in \operatorname{Hom}_{\mathbb{Z}_{p}^{\wedge} G}\left(\mathbb{Z}_{p}^{\wedge} G / H, \mathbb{Z}_{p}^{\wedge} G / H\right) .
$$

Then, arguing as the proof of our main theorem, we see that $M(G / H)$ is a direct summand of $M(n G /\{e\})=M(G /\{e\})^{\oplus n}=0$.

Theorem 5.5. Let $M$ be a cohomotopical Mackey functor over $\mathbb{Z}_{p}^{\wedge}$ such that $M(G /\{e\})=0$. Then, under the same assumption and the notation of Theorem 5.1, we have a formal isomorphism of continuous $\mathbb{Z}_{p}^{\wedge}$-modules:

$$
M(G / G)=\sum_{\sigma \in \triangle / G}(-1)^{\operatorname{dim}(\sigma)} M\left(G / G_{\sigma}\right) .
$$

Proof. This is a direct consequence of Theorem 5.3, the Main Theorem, and Lemma 5.4 .

Similarly, Theorem 5.3, with the help of the Main Theorem, implies the following:

Theorem 5.6. Let $M$ be a cohomotopical Mackey functor over $\mathbb{Z}_{p}^{\wedge}$. Then, under the same assumption and the notation of Theorem 5.3, we have a formal isomorphism of continuous $\mathbb{Z}_{p}^{\wedge}$-modules:

$$
M(G / G)=\sum_{H \in \mathcal{X}} \frac{\mu(H)}{[G: H]} M(G / H) .
$$

\section{Topological applications}

In this section we give some topological applications of our algebraic investigations. We first recall that the Eilenberg-MacLane space $K(M, n)[5,12]$ associated with a Mackey functor $M$ becomes an infinite loop $G$-space and they form an $R O(G)$-graded cohomology theory $\tilde{H}_{G}^{*}(?, M)[22]$. It's easy to see, for any $G$-space $X$ and $* \in R O(G)$, that the functor $S \mapsto \tilde{H}_{G}^{*}\left(X_{+} \wedge S_{+}, M\right)$ becomes a cohomotopical Mackey functor over $\mathbb{Z}_{p}^{\wedge}$ whenever $M$ is. As in the previous section, we assume $G$ is a finite group. Thus we have the following corollaries of Theorems 5.5 and 5.6.

Corollary 6.1. Let $X$ be a $G$-space, $* \in R O(G)$, and $M$ a cohomotopical Mackey functor such that $\tilde{H}_{G}^{*}\left(X_{+} \wedge G /\{e\}_{+}, M\right) \cong H^{*}(X, M(G /\{e\}))=0$, where $\bar{*}$ is the dimension of $*$. Then under the same assumption and the notation of Theorem 5.1, we have a formal isomorphism of continuous $\mathbb{Z}_{p}^{\wedge}$-modules:

$$
\tilde{H}_{G}^{*}\left(X_{+}, M\right)=\sum_{\sigma \in \triangle / G}(-1)^{\operatorname{dim}(\sigma)} \tilde{H}_{G_{\sigma}}^{*}\left(X_{+}, M\right) .
$$

Corollary 6.2. Let $X$ be a $G$-space, $* \in R O(G)$, and $M$ a cohomotopical Mackey functor. Then, under the same assumption and the notation of Theorem 5.3, we have a formal isomorphism of continuous $\mathbb{Z}_{p}^{\wedge}$-modules:

$$
\tilde{H}_{G}^{*}\left(X_{+}, M\right)=\sum_{H \in \mathcal{X}} \frac{\mu(H)}{[G: H]} \tilde{H}_{H}^{*}\left(X_{+}, M\right) .
$$


In particular, when we take $M=\mathbb{Z}_{p}^{\wedge}$, the $\mathbb{Z}_{p}^{\wedge}$-constant Mackey functor [22] [24, v.9.10], we have the following corollaries.

Corollary 6.3. Let a $G$-space $X$ and $i \in \mathbb{N}$ be such that $H^{i}\left(X, \mathbb{Z}_{p}^{\wedge}\right)=0$. Then, under the same assumption and the notation of Theorem 5.1, we have a formal isomorphism of continuous $\mathbb{Z}_{p}^{\wedge}$-modules:

$$
H^{i}\left(X / G, \mathbb{Z}_{p}^{\wedge}\right)=\sum_{\sigma \in \triangle / G}(-1)^{\operatorname{dim}(\sigma)} H^{i}\left(X / G_{\sigma}, \mathbb{Z}_{p}^{\wedge}\right) .
$$

Corollary 6.4. Let $X$ be a $G$-space and $i \in \mathbb{N}$. Then, under the same assumption and the notation of Theorem 5.3, we have a formal isomorphism of continuous $\mathbb{Z}_{p}^{\wedge}$-modules:

$$
H^{i}\left(X / G, \mathbb{Z}_{p}^{\wedge}\right)=\sum_{H \in \mathcal{X}} \frac{\mu(H)}{[G: H]} H^{i}\left(X / H, \mathbb{Z}_{p}^{\wedge}\right)
$$

Actually, these two results are immediate consequences of Yoshida's Theorem [46] (see Theorem 3.7); just check that the classical transfer for the (possibly ramified) covering forms a cohomological Mackey functor (in the original sense).

Theorem 6.5. Let $X$ be a free $G$-space such that $\left(\Sigma^{\infty} X\right)_{p}^{\wedge}$ is contractible. Then under the same assumption and the notation of Theorem 5.1, we have a formal equivalence of spectra:

$$
\left(\Sigma^{\infty} X / G\right)_{p}^{\wedge}=\sum_{\sigma \in \triangle / G}(-1)^{\operatorname{dim}(\sigma)}\left(\Sigma^{\infty} X / G_{\sigma}\right)_{p}^{\wedge}
$$

Proof. By Corollary 3.11, the correspondence

$$
S \mapsto\left(\Sigma^{\infty} X \times_{G} S_{+}\right)_{p}^{\wedge}
$$

is a cohomotopical Mackey functor over $\mathbb{Z}_{p}^{\wedge}$. From the assumption and the usual transfer argument, we see that

$$
\left(\Sigma^{\infty} X \times_{G} G / H_{+}\right)_{p}^{\wedge}=\left(\Sigma^{\infty} X / H_{+}\right)_{p}^{\wedge} \simeq\left(\Sigma^{\infty} S^{0}\right)_{p}^{\wedge}
$$

for any subgroup $H$ of $G$ with $(p,|H|)=1$. So, applying Theorem 5.1 and the Main Theorem to the above cohomotopical Mackey functor, we see that

$$
\left(\Sigma^{\infty} X / G_{+}\right)_{p}^{\wedge}=\sum_{\sigma \in \triangle / G}(-1)^{\operatorname{dim}(\sigma)}\left(\Sigma^{\infty} X / G_{\sigma_{+}}\right)_{p}^{\wedge},
$$

modulo finitely many wedge sums of $\left(\Sigma^{\infty} S^{0}\right)_{p}^{\wedge}$. However, $\left(\Sigma^{\infty} X / K\right)_{p}^{\wedge}$ is connected for any subgroup $K$ of $G$, by the assumption. Thus the above equivalence implies the equality

$$
\left(\Sigma^{\infty} X / G\right)_{p}^{\wedge}=\sum_{\sigma \in \triangle / G}(-1)^{\operatorname{dim}(\sigma)}\left(\Sigma^{\infty} X / G_{\sigma}\right)_{p}^{\wedge}
$$

as was desired. 
Theorem 6.6. Let $X$ be a free $G$-space, Then, under the same assumption and the notation of Theorem 5.3, we have a formal isomorphism of spectra:

$$
\left(\Sigma^{\infty} X / G\right)_{p}^{\wedge}=\sum_{H \in \mathcal{X}} \frac{\mu(H)}{[G: H]}\left(\Sigma^{\infty} X / H\right)_{p}^{\wedge} .
$$

Proof. As in the proof of Theorem 6.5, we obtain an equality

$$
\left(\Sigma^{\infty} X / G_{+}\right)_{p}^{\wedge}=\sum_{H \in \mathcal{X}} \frac{\mu(H)}{[G: H]}\left(\Sigma^{\infty} X / H_{+}\right)_{p}^{\wedge},
$$

by applying Theorem 5.6 this time. However, it is easy to see that

$$
1=\sum_{H \in \mathcal{X}} \frac{\mu(H)}{[G: H]}
$$

from Theorem 5.3 (cf. [44, Prop.8.4(ii)]). This means we can eliminate the extra base points from the above formal equality of spectra, which completes the proof.

Remark 6.7. For $X=E G$, Theorem 6.6 was called the "Minami-Webb formula" in $[27,28]$. There is a recent interesting preprint of Martino and Priddy [29] on this subject.

We now offer another proof of Theorem 6.5 and Theorem 6.6 without using our Main Theorem; this proof is the simplest and most elementary one for these two results. For this purpose, it suffices to provide an elementary proof of the following fact (which is as a matter of course an immediate consequence of the Main Theorem):

Lemma 6.8. Let $G$ be a (possibly infinite) discrete group, and let $S$ and $T$ be finite G-sets such that

$$
\mathbb{F}_{p} S \cong \mathbb{F}_{p} T
$$

as permutation $\mathbb{F}_{p} G$-modules. Then for any free $G$-space $X$, there is an equivalence of spectra:

$$
\left(\Sigma^{\infty} X \times_{G} S\right)_{p}^{\wedge}=\left(\Sigma^{\infty} X \times_{G} T\right)_{p}^{\wedge}
$$

Proof. From the assumption, there are

$$
f \in \operatorname{Hom}_{\mathbb{F}_{p} G}\left(\mathbb{F}_{p} S, \mathbb{F}_{p} T\right) \text { and } g \in \operatorname{Hom}_{\mathbb{F}_{p} G}\left(\mathbb{F}_{p} T, \mathbb{F}_{p} S\right)
$$

such that

$$
f \circ g=1_{\mathbb{F}_{p} T}, \quad g \circ f=1_{\mathbb{F}_{p} S} .
$$

Then let $f^{\prime} \in \operatorname{Hom}_{\mathbb{Z} G}(\mathbb{Z} S, \mathbb{Z} T)$ and $g^{\prime} \in \operatorname{Hom}_{\mathbb{Z} G}(\mathbb{Z} T, \mathbb{Z} S)$ be arbitrary lifts of $f \in \operatorname{Hom}_{\mathbb{F}_{p} G}\left(\mathbb{F}_{p} S, \mathbb{F}_{p} T\right)$ and $g \in \operatorname{Hom}_{\mathbb{F}_{p} G}\left(\mathbb{F}_{p} T, \mathbb{F}_{p} S\right)$, respectively.

We now claim that lifts $\tilde{f} \in \operatorname{Mor}_{\mathcal{M}}(S, T)$ and $\tilde{g} \in \operatorname{Mor}_{\mathcal{M}}(T, S)$ of $f^{\prime}$ and $g^{\prime}$ respectively exist, and that $\tilde{f}$ and $\tilde{g}$ respectively induce the corresponding spectra maps

$$
\begin{aligned}
& F: \Sigma^{\infty} X \times_{G} S_{+} \rightarrow \Sigma^{\infty} X \times_{G} T_{+}, \\
& G: \Sigma^{\infty} X \times_{G} T_{+} \rightarrow \Sigma^{\infty} X \times_{G} S_{+} .
\end{aligned}
$$

Although the existence of such lifts and the resulting spectra maps are more or less immediate consequences of Corollary 2.36 and Exampe 3.3(ii), we now make a 
little detour by constructing such lifts and spectra maps explicitly for the reader's convenience, especially for those readers who are simply interested in this result on spectra and have decided to skip the cohomotopical development.

For this purpose, we first set some notation. Given a sequence of finite index subgroups $K \subseteq H \subseteq G$ and an element $g \in G$, we let

$$
\begin{gathered}
i_{K}^{H}:\left(X \times_{G} G / K\right)_{+} \rightarrow\left(X \times_{G} G / H\right)_{+}, \\
c_{g}:\left(X \times_{G} G / K^{g}\right)_{+} \rightarrow\left(X \times_{G} G / K\right)_{+}
\end{gathered}
$$

denote maps induced by the inclusion $K \subseteq H$ and the conjugation $K^{g} \rightarrow K ; g^{-1} k g$ $\rightarrow k$, respectively. Furthermore, we let

$$
t_{K}^{H}: \Sigma^{\infty}\left(X \times{ }_{G} G / H\right)_{+} \rightarrow \Sigma^{\infty}\left(X \times{ }_{G} G / K\right)_{+}
$$

denote the stable transfer map induced by the inclusion $K \subseteq H$. Notice that these maps respectively correspond to $\operatorname{Res}_{K}^{H}, c_{g}, \operatorname{Ind}_{K}^{H}$ in Definition 2.2.

Next, let $L$ and $M$ be two finite index subgroups of $G$. Through the isomorphisms

$$
\begin{aligned}
& \operatorname{Mor}_{\mathcal{H}}(G / L, G / M)=\operatorname{Hom}_{\mathbb{Z} G}(\mathbb{Z} G / L, \mathbb{Z} G / M) \\
& \cong \operatorname{Hom}_{\mathbb{Z} L}(\mathbb{Z}, \mathbb{Z} G / M) \cong(\mathbb{Z} G / M)^{L} \cong \mathbb{Z}[L \backslash G / M],
\end{aligned}
$$

we see that $\operatorname{Mor}_{\mathcal{H}}(G / L, G / M)$ is a free abelian group with elements of the form $L x M \in \mathbb{Z} L \backslash G / M$ as its basis. Furthermore, we see that this basis element $L x M$ corresponds to

$$
\left(g L \mapsto g \sum_{l \in L / L \cap M^{x^{-1}}} l x M\right) \in \operatorname{Hom}_{\mathbb{Z} G}(\mathbb{Z} G / L, \mathbb{Z} G / M)
$$

through the above isomorphism. But it follows easily from Definition 2.3 that this element is the Hurewicz functor image of

$$
c_{x^{-1}} \circ \operatorname{Res}_{L \cap M^{x^{-1}}}^{M^{x^{-1}}} \circ \operatorname{Ind}_{L \cap M^{x^{-1}}}^{L} \overbrace{G / L}^{G / L \cap M^{x^{-1}}}] \in \operatorname{Mor}_{\mathcal{M}}(G / L, G / M),
$$

where $p$ is the obvious canonical projection and $r p: G / L \cap M^{x^{-1}} \rightarrow G / M$ is the composite of the canonical projection onto $G / M^{x^{-1}}$ and $r_{x}: G / M^{x^{-1}} \rightarrow$ $G / M ; g M^{x^{-1}}=g x M x^{-1} \mapsto g x M$, the right multiplication by $x$.

Motivated by this observation, we define a map

$$
\begin{aligned}
\alpha_{X}(G / L, G / M): \operatorname{Hom}_{\mathbb{Z} G} & (\mathbb{Z} G / L, \mathbb{Z} G / M) \\
& \rightarrow\left\{\Sigma^{\infty}\left(X \times \times_{G} G / L\right)_{+}, \Sigma^{\infty}\left(X \times{ }_{G} G / M\right)_{+}\right\}
\end{aligned}
$$

by

$$
L x M \mapsto c_{x^{-1}} \circ i_{L \cap M^{x^{-1}}}^{M^{x^{-1}}} \circ t_{L \cap M^{x^{-1}}}^{L}
$$


To generalize this definition, recall the canonical decompositions:

$$
\begin{gathered}
\operatorname{Hom}_{\mathbb{Z} G}(\mathbb{Z} S, \mathbb{Z} T)=\bigoplus_{i, j} \operatorname{Hom}_{\mathbb{Z} G}\left(\mathbb{Z} G / L_{i}, \mathbb{Z} G / M_{j}\right), \\
\left\{\Sigma^{\infty}\left(X \times_{G} S\right)_{+}, \Sigma^{\infty}\left(X \times_{G} T\right)_{+}\right\} \\
=\bigoplus_{i, j}\left\{\Sigma^{\infty}\left(X \times_{G} G / L_{i}\right)_{+}, \Sigma^{\infty}\left(X \times_{G} G / M_{j}\right)_{+}\right\},
\end{gathered}
$$

where $S=\coprod_{i} G / L_{i}, T=\coprod_{j} G / M_{j}$.

Then the above definition is generalized to

$$
\alpha_{X}(S, T): \operatorname{Hom}_{\mathbb{Z} G}(\mathbb{Z} S, \mathbb{Z} T) \rightarrow\left\{\Sigma^{\infty}\left(X \times_{G} S\right)_{+}, \Sigma^{\infty}\left(X \times_{G} T\right)_{+}\right\},
$$

by setting the $(i, j)$-component to be $\alpha_{X}\left(G / L_{i}, G / M_{j}\right)$.

Having done this, we may simply set

$$
\begin{aligned}
& F=\alpha_{X}(S, T)(\tilde{f}): \Sigma^{\infty} X \times_{G} S_{+} \rightarrow \Sigma^{\infty} X \times_{G} T_{+}, \\
& G=\alpha_{X}(T, S)(\tilde{f}): \Sigma^{\infty} X \times_{G} T_{+} \rightarrow \Sigma^{\infty} X \times_{G} S_{+},
\end{aligned}
$$

which finishes our little detour.

Now we are ready to finish the proof. Although

$$
F \circ G \neq 1_{\Sigma \infty X \times{ }_{G} T_{+}}, \quad G \circ F \neq 1_{\Sigma \infty X \times{ }_{G} S_{+}}
$$

in general, they are the same at the level of mod-p homology. In fact, this follows from (6.1) by noticing that the correspondence

$$
S \mapsto \tilde{H}_{*}\left(\Sigma^{\infty} X \times_{G} S_{+} ; \mathbb{F}_{p}\right)
$$

defines a cohomological Mackey functor over $\mathbb{F}_{p}$. Of coures, this completes the proof.

Remark 6.9. 1. The reader might wonder why we should consider all the mod-p cyclic subgroups even though the mod- $p$ cohomology of a group is detected by its $p$-Sylow subgroup. Now the following example by [35] negatively answers this question:

$$
B(\mathbb{Z} / 2 \times \mathbb{Z} / 2) \simeq B A_{4} \vee L(2) \vee L(2) \vee B \mathbb{Z} / 2 \vee B \mathbb{Z} / 2 \text {, as 2-local spectra. }
$$

In this example, no matter how we try to express $B A_{4}$ using 2-subgroups $\mathbb{Z} / 2 \times \mathbb{Z} / 2, \mathbb{Z} / 2$, we can never do it, since we can't handle $L(2)$.

2. In [44], the following formal isomorphism as $\mathbb{Z}_{2}^{\wedge} \Sigma_{4}$-modules was given as an example:

$$
\mathbb{Z}_{2}^{\wedge} \Sigma_{4} / \Sigma_{4}=\frac{1}{2} \mathbb{Z}_{2}^{\wedge} \Sigma_{4} / A_{4}+\mathbb{Z}_{2}^{\wedge} \Sigma_{4} / D_{8}-\frac{1}{2} \mathbb{Z}_{2}^{\wedge} \Sigma_{4} /(\mathbb{Z} / 2)^{2} .
$$

This is consistent with the following 2-local stable splittings due to [35]:

$$
\begin{aligned}
& B \Sigma_{4} \simeq B P S L_{2}\left(\mathbb{F}_{7}\right) \vee L(2) \vee B \mathbb{Z} / 2, \\
& B D_{8} \simeq B P S L_{2}\left(\mathbb{F}_{7}\right) \vee L(2) \vee L(2) \vee B \mathbb{Z} / 2 \vee B \mathbb{Z} / 2,
\end{aligned}
$$

which together imply

$$
B \Sigma_{4} \simeq \frac{1}{2} B A_{4}+B D_{8}-\frac{1}{2} B(\mathbb{Z} / 2)^{2} .
$$

This coincidence led Stewart Priddy to conjecture Theorem 6.6 for $X=$ $E G$, which in turn became the starting point of this research. 
3. In view of [33], our main theorem may be applied to study the classifying spaces of more general infinite discrete groups.

4. While our results do not say anything about (non-finite) compact Lie groups, the Mackey functor (and even the Hecke category) for compact Lie groups was defined and studied in $[24,43,21]$. Of course, the problem is how to define the cohomotopical Mackey category in this context (cf. [43]) and (if so) wheather it is useful (like the finite group case or not). As a related subject, [19] and [34] may be regarded as studies of the so-called Burnside category for compact Lie groups.

\section{REFERENCES}

1. J. F. Adams, On the structure and applications of the Steenrod algebra, Comment. Math. Helvetici 32 (1958), 180-214. MR 20:2711

2. J. F. Adams, Prerequisites (on equivariant theory) for Carlsson's lecture, Lecture Notes in Math. 1051 Springer-Verlag, Berlin-New York. 1984, 483-532. MR 86f:57037

3. A. Adem, R. L. Cohen and W. G. Dwyer, Generalized Tate homology, homotopy fixed points and the transfer, Contemporary Mathematics 96, American Mathematical Society (1989), 1-13. MR 90k:55012

4. F. W. Anderson and K. R. Fuller, Rings and Categories of Modules, 2nd ed., Graduate Texts in Mathematics 13, Springer-Verlag, New York-Berlin-Heidelberg (1992). MR 94i:16001

5. G. E. Bredon, Equivariant cohomology theories, Lecture Notes in Math. 34 Springer-Verlag, Berlin-New York. (1967). MR 35:4914

6. G. Carlsson, Equivariant stable homotopy and Segal's Burnside ring conjecture, Annals of Math. 120 (1984), 189-224. MR 86f:57036

7. E. Cline, B. Parshall and L. Scott, Cohomology of finite groups of Lie type, I, Publ. Math. I.H.E.S. 45 (1975) 169-191. MR 53:3134

8. S. B. Conlon, Decompositions induced from the Burnside Algebra, J.Algebra 10 (1968), 102122. MR 38:5945

9. C. Curtis and I. Reiner, Representation theory of finite groups and associative algebras, Interscience Publishers, New York (1962). MR 26:2519

10. A. Dress, Contributions to the theory of induced representations, Lecture Notes in Math. 342 Springer-Verlag, Berlin-New York. 1973, 183-240. MR 52:5787

11. A. Dress, Induction and structure theorems for orthogonal representations of finite groups, Annals of Math. 102 (1975), 291-325. MR 52:8235

12. A. Elmendorf, Systems of fixed point sets, Trans. Amer. Math. Soc. 277 (1983), 275-284. MR 84f: 57029

13. D. Gluck, Idempotent formula for the Burnside algebra with applications to the p-subgroup simplicial complex, Ill. J. Math. 25 (1981), 63-67. MR 82c:20005

14. J. A. Green, Axiomatic representation theory for finite groups, J. Pure and Applied Algebra 1 (1971), 41-77. MR 43:4931

15. J. P. C. Greenlees, Representing Tate cohomology of G-spaces, Proc. Edinburgh Math. Soc. 30 (1987), 435-443. MR 88m:57055

16. J. P. C. Greenlees and J. P. May, Generalized Tate cohomology, Mem. Amer. Math. Soc. 113 (1995), no. 543, viii+178 pp. MR 96e:55006

17. C. Kosniowski, Equivariant cohomology and stable cohomotopy, Math. Ann. 210 (1974), 83104. MR 54:1202

18. E. Laitinen, On the Burnside ring and stable cohomotopy of a finite group, Math. Scand. 44 (1979), 37-72. MR 80k:55030

19. C-N. Lee and N. Minami, Segal's Burnside ring conjecture for compact Lie groups, pp.133161, Algebraic topology and Its Applications (1994) Springer-Verlag. MR 95b:55001

20. L. G. Lewis, Jr., An introduction to Mackey functors, a draft.

21. L. G. Lewis, Jr., The Category of Mackey Functors for a Compact Lie Group, Group Representations: Cohomology, Group Actions and Topology, Proc. Sympos. Pure Math., vol. 63, Amer. Math. Soc., Providence, RI, 1998, pp. 301-354. CMP 98:08

22. L. G. Lewis, Jr., J. P. May and J. E. McClure, Ordinary RO(G)-graded cohomology, Bull. Amer. Math. Soc. 4 (1981), 208-212. MR 82e:55008 
23. L. G. Lewis, Jr., J. P. May and J. E. McClure, Classifying G-spaces and the Segal conjecture, Canadian Math. Soc. Conference Proceedings Vol 2, part 1 (1982), 165-179. MR 84d:55007a

24. L. G. Lewis, Jr., J. P. May and M. Steinberger (with contributions by J. E. McClure), Equivariant Stable Homotopy Theory, Lecture Notes in Math. 1213 Springer-Verlag, Berlin-New York. 1986. MR 88e:55002

25. H. Linder, A remark on Mackey functors, Manuscripta Math. 18 (1976), 273-278. MR 53:5691

26. F. Luca, The algebra of Green and Mackey functors, The University of Alaska Fairbanks Ph. D. Thesis. (1996).

27. J. Martino and S. Priddy, A classification of the stable type of BG, Bull. Amer. Math. Soc. (N.S.) 27 (1992), 165-170. MR 93b:55019

28. J. Martino and S. Priddy, The complete stable splitting for the classifying space of a finite group, Topology 31 (1992), 143-156. MR 93d:55012

29. J. Martino and S. Priddy, Applications of the Minami-Webb formula to splitting classifying spaces, preprint.

30. J. P. May, Stable maps between classifying spaces, Contemporary Mathematics 37 (1985), 121-129. MR 86m:55022

31. N. Minami, On the $I(G)$-adic topology of the Burnside rings of compact Lie groups, Publ. Res. Inst. Math. Sci. 20 (1984), 447-460. MR 86g:57029

32. N. Minami, "A Hecke Algebra" for cohomotopical Mackey functors, the stable homotopy of orbit spaces, and a Möbius function, MSRI preprint series 00425-91 (1990).

33. N. Minami, On the classifying spaces of $S L_{3}(\mathbb{Z}), S t_{3}(\mathbb{Z})$ and finite Chevalley groups, Contemp. Math., vol. 158 (1994), 175-185. MR 95b:55015

34. N. Minami, The relative Burnside module and stable maps between classifying spaces of compact Lie groups, Trans. Amer. Math. Soc., 347 (1995), 461-498. MR 95i:55008

35. S. Mitchell and S. Priddy, Symmetric product spectra and splittings of classifying spaces, Amer. J. Math. 106 (1984), 219-232. MR 86g:55009

36. G. Nishida, The nilpotency of elements of the stable homotopy groups of spheres, J. Math. Soc. Japan 25 (1973), 707-732. MR 49:6236

37. S. Priddy, Recent progress in stable splittings, London Mathematical Society Lecture Notes Series 117, 149-174, Cambridge University Press 1987. MR 89f:55017

38. D. Quillen, Homotopical algebra, Lecture Notes in Math. 43 Springer-Verlag, Berlin-New York. 1967. MR 36:6480

39. D. Quillen, Homotopy properties of the poset of nontrivial p-subgroups of a group, Advances in Math. 28 (1978), 101-128. MR 80k:20049

40. G. Shimura, Introduction to the arithmetic theory of automorphic functions, Iwanami Shoten and Princeton University Press, 1971. MR 47:3318

41. J. Thévenaz and P. Webb, The structure of Mackey functors, Trans. Amer. Math. Soc. 347 (1995), 1865-1961. MR 95i:22018

42. R. W. Thomason, The homotopy limit problem, Contemporary Mathematics 19, American Mathematical Society 1983. MR 84j:18012

43. S. Waner, Mackey functors and G-cohomology, Proc. Amer. Math. Soc., 85 (1982), 641-648. MR 85e: 55013

44. P. J. Webb, A Local Method in Group Cohomology, Comment. Math. Helvetici 62 (1987), 135-167. MR 88h:22065

45. K. Wirthmüller, Equivariant homology and duality, Manuscripta Math. 11 (1974), 373-390. MR 49:8004

46. T. Yoshida, On G-functors (II): Hecke operators and G-functors, J. Math. Soc. Japan 35 (1983), 179-190. MR 84b:20010

47. T. Yoshida, Idempotents of the Burnside rings and Dress induction theorem, J. Algebra 80 (1983), 90-105. MR 85d:20004

Department of Mathematics, The University of Alabama, Box 870350, Tuscaloosa Alabama 35487-0350

E-mail address: norihiko@gp.as.ua.edu 